\title{
Towards a topological quantum chemistry description of correlated systems: The case of the Hubbard diamond chain
}

\author{
Mikel Iraola $\odot,{ }^{1,2, *}$ Niclas Heinsdorf, ${ }^{3}$ Apoorv Tiwari $\odot,{ }^{4,5}$ Dominik Lessnich $\odot,{ }^{3}$ Thomas Mertz $\odot,{ }^{3}$ Francesco Ferrari $\odot,{ }^{3}$ \\ Mark H. Fischer $\odot,{ }^{5}$ Stephen M. Winter, ${ }^{3,6}$ Frank Pollmann, ${ }^{7}$ Titus Neupert $\odot,{ }^{5}$ Roser Valentí $\odot,{ }^{3, \dagger}$ and Maia G. Vergniory ${ }^{1,8, \hbar}$ \\ ${ }^{1}$ Donostia International Physics Center, 20018 Donostia-San Sebastian, Spain \\ ${ }^{2}$ Department of Physics, University of the Basque Country UPV/EHU, Apartado 644, 48080 Bilbao, Spain \\ ${ }^{3}$ Institute of Theoretical Physics, Goethe University Frankfurt, Max-von-Laue-Straße 1, 60438 Frankfurt am Main, Germany \\ ${ }^{4}$ Condensed Matter Theory Group, Paul Scherrer Institute, CH-5232 Villigen PSI, Switzerland \\ ${ }^{5}$ Department of Physics, University of Zurich, Winterthurerstrasse 190, CH-8057 Zurich, Switzerland \\ ${ }^{6}$ Department of Physics, Wake Forest University, 1834 Wake Forest Road, Winston-Salem, North Carolina 27109-7507, USA \\ ${ }^{7}$ Department of Physics and Institute for Advanced Study, Technical University of Munich, 85748 Garching, Germany \\ ${ }^{8}$ IKERBASQUE, Basque Foundation for Science, Maria Diaz de Haro 3, 48013 Bilbao, Spain
}

(Received 13 February 2021; accepted 21 October 2021; published 15 November 2021)

\begin{abstract}
The recently introduced topological quantum chemistry (TQC) framework has provided a description of universal topological properties of all possible band insulators in all space groups based on crystalline unitary symmetries and time reversal. While this formalism filled the gap between the mathematical classification and the practical diagnosis of topological materials, an obvious limitation is that it only applies to weakly interacting systems, which can be described within band theory. It is an open question to which extent this formalism can be generalized to correlated systems that can exhibit symmetry-protected topological phases which are not adiabatically connected to any band insulator. In this work, we address the many facets of this question by considering the specific example of an extended version of a Hubbard diamond chain. This model features a Mott insulator, a trivial insulating phase, and an obstructed atomic limit phase. Here we first discuss the nature of the Mott insulator and determine the phase diagram and topology of the interacting model with infinite density matrix renormalization group calculations, variational Monte Carlo simulations, and with many-body topological invariants. We then proceed by considering a generalization of the TQC formalism to Green's functions combined with the concept of a topological Hamiltonian to identify the topological nature of the phases. Here we use cluster perturbation theory to calculate the Green's functions. The results are benchmarked with the above-determined phase diagram, and we discuss the applicability and limitations of the approach and its possible extensions in the diagnosis of topological phases in materials, in contrast to the use of many-body topological invariants.
\end{abstract}

DOI: 10.1103/PhysRevB.104.195125

\section{INTRODUCTION}

Topology is one of the central concepts in the modern understanding of electronic quantum matter [1-3]. One of its first incarnations, the quantum Hall effect [4], demonstrates impressively that topological phenomena can be intimately linked, yet vastly different, depending on whether or not electron-electron interactions are required for them to exist: The integer quantum Hall effect can be understood from noninteracting electrons, while its fractional counterpart $[5,6]$ is intrinsically interacting.

This division between the interacting and noninteracting view on topology got reinforced as the characterization and classification of topological phases advanced substantially over the past years. In particular, the role of symmetries, both of spatial (crystalline) and of global type (e.g., time reversal), has been explored in great depth in both domains [7-11].

\footnotetext{
*mikel.i.iraola@gmail.com

${ }^{\dagger}$ valenti@itp.uni-frankfurt.de

${ }^{\ddagger}$ maiagvergniory@dipc.org
}

For interacting phases, the notion of symmetry-protected topology (SPT) [12-18] is central. These phases are often defined from a quantum circuit perspective: Two SPT quantum states are distinct if there is no finite depth circuit of local and symmetry-respecting unitary operators that transform into one another. A trivial SPT state is a direct product of local basis states. Spatial, i.e., nonlocal symmetries have been incorporated in this framework as well. The classification is then derived from that of subdimensional systems that are left invariant under the symmetry, such as a point under inversion symmetry or a plane in the case of three-dimensional mirror symmetry [19]. With this, the understanding of SPT phases arises from a local perspective on the physical system, furnished by the degrees of freedom that form the trivial product state, and they are acted upon by sequences of local unitary operations. In general, the use of many-body invariants based on cobordism theory [20], or, for some specific cases as described above, their formulation in terms of invariants that can be extracted directly from the ground-state wave function, provides a powerful framework to classify SPT phases for interacting fermionic systems [18]. A major drawback 
of this approach, however, is its impracticability to diagnose topological phases in real systems due to the complicated mathematical treatment of the invariants beyond the simplest cases.

Actually, the notion of SPTs applies in principle also in the absence of interactions, but a much more efficient way of detecting and classifying topology of noninteracting electron systems arises from band theory. Two band insulators are topologically distinct if they cannot be smoothly deformed into one another without breaking a set of protecting symmetries or closing the band gap [2,21]. A wealth of topological invariants can be defined from the fiber bundles of Bloch functions over the Brillouin zone (BZ) to detect topological distinctions [22,23]. Examples include Chern numbers for quantum Hall states, winding numbers, and Pfaffian invariants for topological insulators. When spatial symmetries are also taken into account, this classification is refined and topological invariants can be formulated from the irreducible symmetry group representations of the Bloch wave functions of occupied bands [24,25].

Recently, topological quantum chemistry (TQC) emerged as a new perspective on the topology of noninteracting electronic states $[7,26]$. Different from topological band theory, it starts from a real-space description with the realization that there is not only one atomic limit that serves as a trivial reference state, but potentially there are many distinct ones that depend on the symmetries considered. Each of these atomic limits induces a band structure with specific irreducible representations (irreps) of their Bloch states [27-29]. The logic proceeds then by enumerating all these atomic limits and declaring as topological any band structure with its irreps that cannot be built from such atomic limits. Thus, TQC brought a real-space perspective-which was always foundational for studying SPT phases - to noninteracting topological systems.

The purpose of this work is to explore the applicability and practicability of the TQC framework to diagnose interacting SPT phases in real materials, in contrast to the use of manybody invariants. For that, we consider the TQC standpoint that topologically trivial phases of matter can be built from atomic limits, and we first discuss the concept of a Mott SPT phase $[30,31]$ and, in general, Mott phases. In a second step, inspired by TQC and symmetry-based indicators [7,9], we make use of band representations of the single-particle Green's function to investigate and detect certain interacting topological phases. For this purpose, we invoke the concept of a topological Hamiltonian [32-34] defined via the single-particle Green's function, which we calculate here within cluster perturbation theory (CPT) [35-38]. As a proof-of-principle we demonstrate these ideas on the example of a Hubbard model of spinful fermions on an extended version of a diamond chain for which we can determine the phase diagram via infinite density matrix renormalization group (iDMRG) calculations $[39,40]$ complemented by variational Monte Carlo (VMC) simulations [41-44]. The simplicity of the model allows for calculations of many-body wave function topological invariants based on partial reflection operations [45], which serve as a reference for testing the Green's-function-based topological classification of phases. The analysis of the results shows that, in contrast to the mathematical difficulties in the calculation of many-body invariants when considering models beyond the simplest ones, the TQC Green's-function-based approach may be a candidate to become a systematic method to characterize the topology of interacting insulating phases in material-specific models, at least for the cases when they are adiabatically connected to noninteracting phases. We also discuss explicitly the case of the SPT Mott phases.

The paper is organized as follows: In Sec. II, we introduce the Hubbard diamond chain Hamiltonian that we use as a testbed model to study the applicability of TQC to interacting systems. In the example of the noninteracting case, we review in Sec. III the notion of topological classification through elementary band representations (EBRs) rooted in TQC. To be able to use the interacting model as the benchmark of the TQC Green's-function-based approach, in Sec. IV we determine the phase diagram for the Hubbard diamond chain at halffilling via iDMRG and VMC for various values of the on-site Hubbard interaction, and we explore the appearance of a Mott SPT phase. We take advantage of the distinct nature of the two approaches to delineate the phase boundaries. We conclude this section with the computation of many-body topological invariants for the ground-state wave functions that identify the topological nature of the interacting phases. Section V presents the classification of topological phases through an EBR analysis of the single-particle Green's functions of the interacting system. Here, we make use of the concept of a topological Hamiltonian that we combine with CPT to obtain our results and compare with the results obtained in Sec. IV. Finally, in Sec. VI we present our conclusions and outlook on possible extensions and applicability of the TQC Green's-function-based approach in the diagnosis of topological phases in materials, in contrast to the use of many-body topological invariants.

\section{EXTENDED HUBBARD DIAMOND CHAIN}

As illustrated in Fig. 1(a), the Hubbard diamond chain (HDC) consists of a one-dimensional periodic arrangement (along $x$ ) of diamonds with symmetry described by the space group Pmmm (no. 47), where the lattice sites are at $2 i$ and $2 m$ Wyckoff positions (WPs). We consider $s$-orbitals that induce eight bands in reciprocal space, which have pairwise Kramers' degeneracies (spinful fermions).

The model has three different hopping parameters: (i) an intracell nearest-neighbor hopping $t_{1}$, (ii) an intracell nextnearest-neighbor coupling $t_{2}$, and (iii) an intercell coupling $t_{3}$. On-site electron-electron correlations are included through a Hubbard term whose strength is controlled by the Hubbard parameter $U$. The full Hamiltonian in the absence of spin-orbit coupling is given by

$$
\begin{aligned}
\mathcal{H}= & U \sum_{\alpha, j} n_{\alpha, j, \uparrow} n_{\alpha, j, \downarrow}+\sum_{j, \sigma} \sum_{\alpha, \beta} c_{\alpha, j, \sigma}^{\dagger} \mathbb{T}_{\alpha \beta} c_{\beta, j, \sigma} \\
& -\sum_{\sigma, j}\left(t_{3} c_{1, j, \sigma}^{\dagger} c_{3, j+1, \sigma}+\text { H.c. }\right)+\mu \sum_{\alpha, j, \sigma} c_{\alpha, j, \sigma}^{\dagger} c_{\alpha, j, \sigma},
\end{aligned}
$$

where $c_{\alpha, j, \sigma}^{\dagger}\left(c_{\alpha, j, \sigma}\right)$ creates (annihilates) an electron of spin $\sigma$ at site $\alpha \in\{0,1,2,3\}$ of the cell labeled by $j=1, \ldots, N$, with $N$ the number of unit cells and $\mu$ is the chemical potential, which at $T=0$ matches the Fermi energy and is chosen 

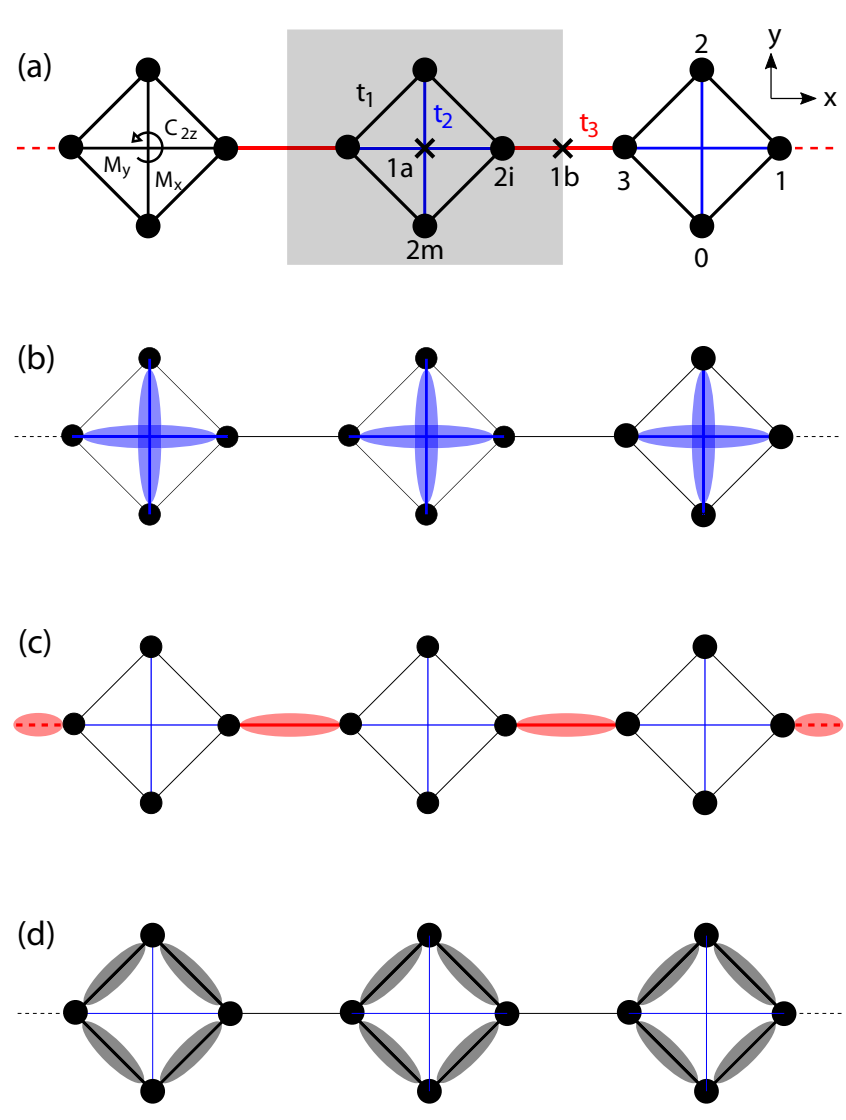

FIG. 1. (a) Atomic configuration of the extended diamond chain. The unit cell is marked with the gray background, WPs $1 a$ and $1 b$ are denoted with crosses, and atomic sites at WPs $2 i$ and $2 m$ are denoted with solid black circles. The enumeration of orbitals adopted to write the Hamiltonian is also shown. Intracell hoppings $t_{1}$ and $t_{2}$ are indicated in black and blue lines, respectively, while intercell hopping $t_{3}$ is indicated in red. Black lines and the circular arrow in the left diamond denote reflection planes and twofold rotation with respect to the $z$-axis, respectively. (b)-(d) Dominant coupling parameters for three different limiting cases: (b) $t_{2} \gg t_{1}, t_{3}$, (c) $t_{3} \gg t_{1}, t_{2}$, and (d) $t_{1} \gg t_{2}, t_{3}$.

such that the system is at half-filling. The matrix $\mathbb{T}_{\alpha \beta}$ that contains the intracell couplings $t_{1}$ and $t_{2}$ has the form

$$
\mathbb{T}=-\left[\begin{array}{cccc}
0 & t_{1} & t_{2} & t_{1} \\
t_{1} & 0 & t_{1} & t_{2} \\
t_{2} & t_{1} & 0 & t_{1} \\
t_{1} & t_{2} & t_{1} & 0
\end{array}\right] .
$$

Actually, the HDC model can be understood as a onedimensional version of the two-dimensional square lattice considered by Yao and Kivelson [30], where the atomic units are Hubbard diamonds.

\section{NONINTERACTING HDC}

In this section, we study the topological nature of the noninteracting HDC within the framework of TQC. For that, we use elementary band representations of the single-valued group Pmmm (the use of the single-valued group is justified by the absence of spin-orbit coupling in the Hamiltonian) to analyze the symmetry representation of bands in each phase.

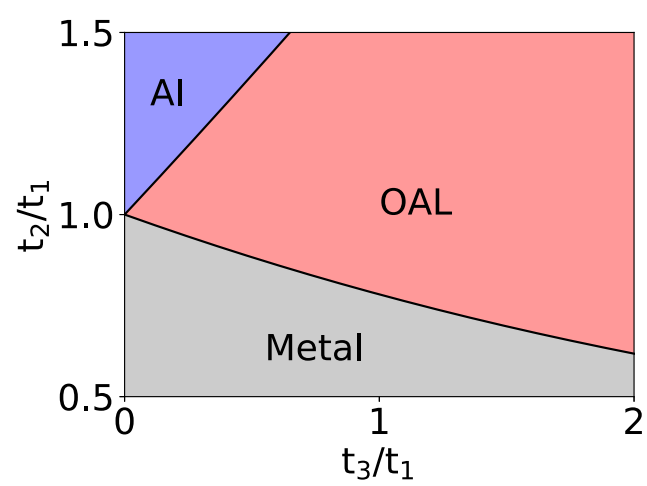

FIG. 2. Phase diagram of the noninteracting diamond chain model at half-filling. The blue represents the AI phase, red the OAL, and gray the metallic phase.

We will follow the notation of the Bilbao Crystallographic Server [46,47].

Our model is induced from orbitals transforming under the $A_{1}$ representation of the point group [48] $C_{2 v}$ [see Fig. 1(a)] on the $2 m$ site, and a second set of orbitals transforming under the same representation on the $2 i$ site. The eight bands in our model thus transform under the composite band representation $\left(A_{1} \uparrow G\right)_{2 i} \oplus\left(A_{1} \uparrow G\right)_{2 m}$. The representations of little groups $G_{k}$ at high symmetry points $\Gamma$ and $X$ of the BZ subduced by this representation can be decomposed as $2 \Gamma_{1}^{+} \oplus \Gamma_{3}^{-} \oplus \Gamma_{4}^{-}$and $2 X_{1}^{+} \oplus X_{3}^{-} \oplus X_{4}^{-}$in terms of irreps.

The analytical phase diagram of the noninteracting $(U=0)$ HDC Hamiltonian (1) for positive $t_{2} / t_{1}, t_{3} / t_{1}$ [49] at half-filling is shown in Fig. 2. The system has a metallic (labeled Metal) and two insulating phases, dubbed atomic insulator (AI) and obstructed atomic limit (OAL).

In the limit $t_{1} \rightarrow 0$, sites at WP $2 i$ and WP $2 m$ are decoupled. The sites at WP $2 m$ form local dimers, while sites at WP $2 i$ are connected along the periodic $x$ direction and form a one-dimensional chain that can be adiabatically connected to the Su-Schrieffer-Heeger (SSH) chain. In particular, when $t_{3} \ll t_{2}$, sites at WP $2 i$ form a chain that can be connected to the trivial SSH chain. This mapping is corroborated by the TQC-based analysis of the band structure: The valence bands transform in the composite band representation $2\left(A_{1} \uparrow G\right)_{1 a}$, with occupied little group representations $2 \Gamma_{1}^{+}$ and $2 X_{1}^{+}$[Fig. 3(a)]. This representation can be induced from two Wannier functions whose charge centers are at the WP $1 a$ and transform like $s$-orbitals under the action of the sitesymmetry group of this site. Since the band representation of the occupied band of the trivial SSH-chain can also be induced by identical Wannier functions, we conclude that the occupied subspace of the diamond chain's spectrum can be adiabatically connected to two copies of the trivial SSH-chain, and we classify this phase as an atomic insulator (AI).

When $t_{3}$ is the dominant hopping term, i.e., $t_{3} \gg t_{1}, t_{2}$ [see Figs. 1(c) and 2], the chain formed by sites at WP $2 i$ can be mapped to the SSH chain in the topological phase. Thus we expect the sites at WP $2 i$ to contribute to the occupied subspace with a band of the same nature. Again, this connection is confirmed from the viewpoint of TQC framework: the valence bands transform in the $\left(A_{1} \uparrow G\right)_{1 a} \oplus\left(A_{1} \uparrow G\right)_{1 b}$ band representation with little group representations $2 \Gamma_{1}^{+}$and $X_{1}^{+} \oplus X_{3}^{-}$. 

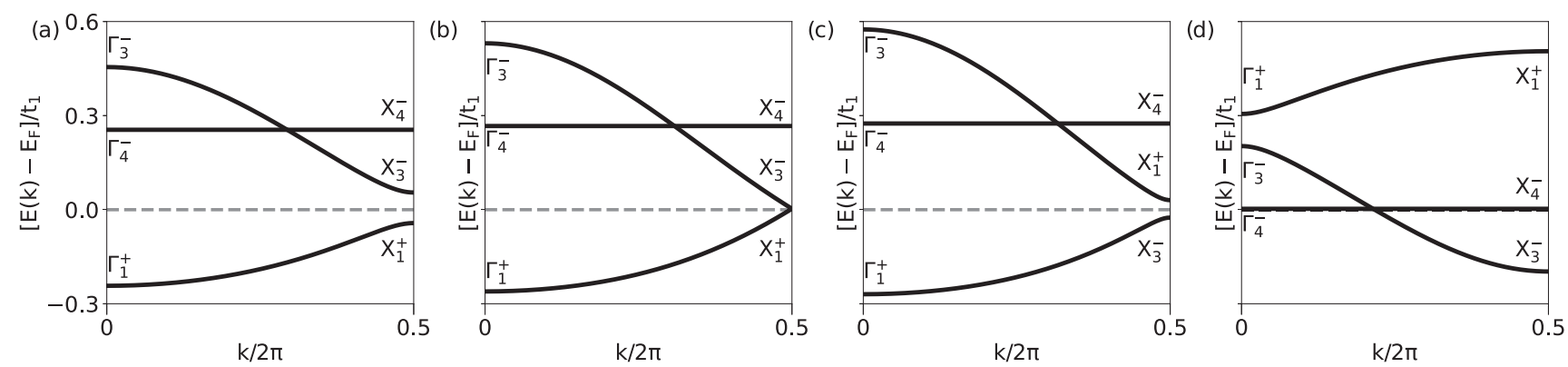

FIG. 3. Band structure of the HDC for $U=0$, where the lowest occupied band has been omitted as it is disconnected from the rest and it contains the irreps $\left\{\Gamma_{1}^{+}, X_{1}^{+}\right\}$in the studied range of parameters [50]. (a) AI phase $\left(t_{2} / t_{1}=1.2, t_{3} / t_{1}=0.2\right)$. (b) Transition point between the AI and OAL phases $\left(t_{2} / t_{1}=1.2, t_{3} / t_{1}=0.264\right)$. (c) OAL phase $\left(t_{2} / t_{1}=1.2, t_{3} / t_{1}=0.3\right)$. (d) Metallic phase $\left(t_{2} / t_{1}=0.8, t_{3} / t_{1}=0.2\right)$.

Particularly, the band with little group irreps $\Gamma_{1}^{+}$and $X_{3}^{-}$can be induced from a Wannier function whose charge center is in the WP $1 b$ and that transforms like an $s$-orbital under the operations of the site-symmetry group. The valence band of the topological SSH-chain is obtained from the subduction of $\left(A_{1} \uparrow G\right)_{1 b}$ to the space group $P \overline{1}$ of the SSH-chain, thus the band with little group irreps $\left\{\Gamma_{1}^{+}, X_{3}^{-}\right\}$in the HDC model can be mapped to the valence band of the topological SSH-chain. On the basis of this mapping, and the fact that the band with irreps $\Gamma_{1}^{+}$and $X_{3}^{-}$is induced from an empty WP, we identify the phase at $t_{3} \gg t_{2}, t_{1}$ as an obstructed atomic limit (OAL).

As can be seen in Figs. 3(a)-3(c), while at $\Gamma$ the irreps are the same for both phases, at $X$ the wave function in the valence band transforms under $X_{1}^{+}$for the AI phase and under $X_{3}^{-}$for the OAL. Since these irreps have different twofold rotation $\hat{C}_{2 z}$ and reflection $\hat{M}_{x}$ [which maps a point $(x, y, z)$ to $(-x, y, z)]$ symmetry eigenvalues, it is not possible to connect these phases by a path in which the gap between valence and conduction bands does not close without breaking these symmetries.

For the last limiting case, where $t_{1}$ is the dominant hopping and $t_{1} \gg t_{2}, t_{3}$ as is shown in Fig. 1(d), the ground state of the single diamond is degenerate. Since $t_{3}$ is negligible, it follows from this degeneracy that the many-body ground state of the HDC chain is also degenerate and therefore metallic, as it is confirmed by the presence of four partially filled bands in the band structure of Fig. 3(d), where the Fermi energy is pinned at the flat band (with little group representations $\Gamma_{4}^{-}$and $X_{4}^{-}$) associated entirely with the $2 m$ sites.

\section{TOPOLOGY OF THE INTERACTING HDC}

\section{A. Phase diagram of $\mathrm{HDC}$ for finite $\boldsymbol{U}$}

We determine the phase diagram of HDC for finite $U$ values via infinite DMRG calculations (iDMRG) as well as variational Monte Carlo (VMC) simulations. The details of the calculations are given in Appendices A and B. Both VMC and DMRG [in the matrix product state (MPS) formulation] are state-of-the-art variational techniques that are employed to approximate the ground-state wave function of quantum many-body Hamiltonians. DMRG is the most prominent numerical method for the study of strongly correlated onedimensional systems, and it has successfully been applied to a large variety of quantum models [51]. The MPS Ansätze can efficiently encode low-entangled quantum states, with systematically improvable accuracy. On the other hand, the VMC method applied in the present work relies on relatively simple variational wave functions, made of a mean-field fermionic state supplemented by Jastrow correlators [41]. Despite its intrinsically biased nature, the VMC method represents a reliable tool for the study of the phase diagram of systems of interacting electrons beyond the perturbative limit, as in the case of Mott transitions in Hubbard-like models.

In Fig. 4, we present the phase diagram extracted from iDMRG simulations for $U / t_{1}=0.4$ and 1 at half-filling by

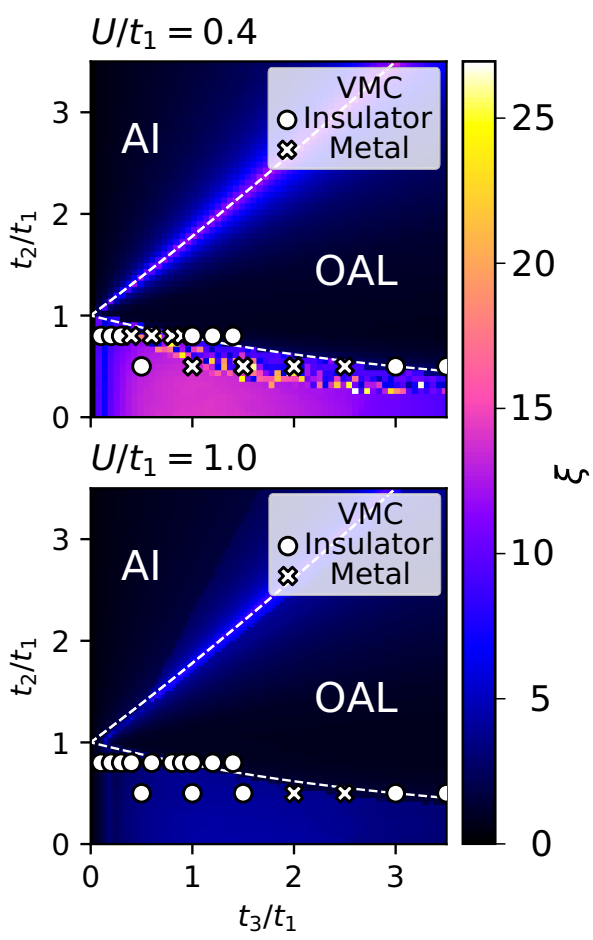

FIG. 4. Phase diagram of the interacting diamond chain for $U / t_{1}=0.4$ and 1 at half-filling determined from the calculation of the correlation length $\xi$ in iDMRG (color map), as well as data points obtained by VMC at $t_{2} / t_{1}=0.8$ and 0.5 for various $t_{3} / t_{1}$ indicating whether the system is in a metallic (crosses) or an insulating (circles) phase. The phase boundaries of the noninteracting phase diagram are given by the white, dashed lines. 
calculating the correlation length $\xi$ as defined in Eq. (A2) of Appendix A. Phase diagrams for $U / t_{1}=2$ and 4 are also shown in Appendix A. While gapped phases are characterized by a finite correlation length, critical points as well as metallic phases have a diverging $\xi$ [52]. Although formally the correlation length $\xi$ diverges at the phase boundaries, it only assumes a large finite value in our data since it is bounded by the maximal bond dimension, which is set to $\chi=128$. For metallic or close-to-metallic systems, iDMRG performs generally poorly due to the large entanglement, resulting in points that are not fully converged close to the lower phase boundary for $U / t_{1}=0.4$ in Fig. 4, upper panel. We have therefore performed VMC simulations as well in order to corroborate the iDMRG results in the region where the metallic phase is observed $\left(t_{2} / t_{1}<1\right)$. Our variational approach is based on Jastrow-Slater wave functions as described in Appendix B. The regions of gapped (insulator) and gapless (metallic) phases as determined by VMC are shown in Fig. 4 as circles and crosses, respectively.

For $t_{2} / t_{1}<1, t_{3} / t_{1} \ll 1$, and any finite $U$, we find a gapped symmetry-preserving Mott-insulating (MI) phase. Increasing $t_{3} / t_{1}$, the system either undergoes a transition into an intermediate metallic phase, or, for sufficiently large values of $U$, it enters the OAL phase directly from the MI phase (compare the results for $U / t_{1}=0.4$ and 1 in the region $t_{2} / t_{1}=0.8$ and $0<t_{3} / t_{1}<1$ in Fig. 4). As we elaborate in Sec. IV B, the MI is a SPT phase that cannot be adiabatically connected to any noninteracting atomic limit provided mirror and rotation symmetries remain unbroken. With increasing $U$, the MI replaces an increasing proportion of the metallic region of the noninteracting model, while the extent of the bordering OAL phase remains largely unchanged.

For $t_{2} / t_{1}>1$ and finite interaction $U$, the transition from the AI to OAL remains but shifts slightly to larger values of $t_{3} / t_{1}$ when increasing $U / t_{1}$ (see also Fig. 9 of Appendix A). Since all single-particle bands are either completely filled or empty in the gapped $\mathrm{AI}$ and OAL phases at $U=0$, a small finite $U$ only induces a renormalization of the electron bands without any drastic change. In analogy with the spinful SSH chain [45], both the AI and OAL phases are also smoothly connected to gapped valence-bond analogs appearing at large $U$, without a change of ground-state symmetry. As a result, the interacting analogs of the $\mathrm{AI}$ and $\mathrm{OAL}$ phases are each smoothly connected to a noninteracting atomic limit.

\section{B. Mott SPT phase and many-body invariants}

In this section, we establish the MI phase as an SPT phase, and we analyze the topology of the interacting phases in the HDC through many-body invariants.

The MI phase may be distinguished from the rest by the properties of the many-body ground-state wave function $\left|\Psi_{0}\right\rangle$ with respect to the crystalline symmetries. $\left|\Psi_{0}\right\rangle$ must transform as an irrep of the space group, which cannot change without gap closure. It is therefore sufficient to consider specific points in the phase diagram to elucidate the ground-state symmetry of each phase.

Let us focus first on the AI and OAL phases. Consider an operator $c_{i}^{\dagger}$ that creates a particle in a state that belongs to the spectra of the single-particle Hamiltonian and $h \in P m m m$, with eigenvalue $\lambda_{h, i}$. Due to time reversal symmetry (TRS) $\mathcal{T}$, the operator $\mathcal{T} c_{i}^{\dagger} \mathcal{T}^{-1}$ corresponds to an energetically degenerate eigenstate of $h$ with eigenvalue $\lambda_{h, i}^{*}$. To obtain a many-body gapped state, these two levels must be either both unoccupied or both occupied. However, the product of operators always transforms trivially:

$$
U_{h} c_{i}^{\dagger}\left(\mathcal{T} c_{i}^{\dagger} \mathcal{T}^{-1}\right) U_{h}^{-1}=\left|\lambda_{h, i}\right|^{2} c_{i}^{\dagger}\left(\mathcal{T} c_{i}^{\dagger} \mathcal{T}^{-1}\right),
$$

since $\left|\lambda_{h, i}\right|^{2}=1$, where $U_{h}$ is the representation of $h$. In other words, any state described by a single Slater determinant transforms trivially provided all single-particle levels are either empty or fully occupied with both spin up and spin down. This condition is satisfied by all states that can be adiabatically connected to a noninteracting gapped state of spinful particles with time-reversal symmetry, in particular the $\mathrm{AI}$ and $\mathrm{OAL}$ phases.

An alternative way to show that the ground state of the AI phase transforms as the trivial representation of Pmmm follows by considering the limit $t_{3} \rightarrow 0$. In this case, $\left|\Psi_{0}\right\rangle$ is a product state of the local ground states of each diamond. Let us denote with $O_{j}^{\dagger}$ the operator that creates the ground state of the $j$ th diamond. Then

$$
\left|\Psi_{0}\right\rangle=O_{1}^{\dagger} \otimes O_{2}^{\dagger} \otimes \cdots \otimes O_{N}^{\dagger}|0\rangle \equiv \bigotimes_{j} O_{j}^{\dagger}|0\rangle,
$$

where $j$ runs over all diamonds (unit cells) and $|0\rangle$ denotes the vacuum state. Since $O_{j}^{\dagger}$ contain four fermionic operators, it follows that they commute on different diamonds: $\left[O_{i}^{\dagger}, O_{j}^{\dagger}\right]=$ 0 . This provides that $\left|\Psi_{0}\right\rangle$ transforms trivially under discrete lattice translation:

$$
T_{x}\left|\Psi_{0}\right\rangle=O_{2}^{\dagger} \otimes \cdots \otimes O_{N}^{\dagger} \otimes O_{1}^{\dagger}|0\rangle=\left|\Psi_{0}\right\rangle,
$$

where periodic boundary conditions have been assumed. At $t_{2} / t_{1} \gg 1$ and $t_{3}=U=0$, the operator $O_{j}^{\dagger}$ is given by

$$
O_{\mathrm{AI}, j}^{\dagger}=\tilde{c}_{\pi, j, \uparrow}^{\dagger} \tilde{c}_{\pi, j, \downarrow}^{\dagger} \tilde{c}_{0, j, \uparrow}^{\dagger} \tilde{c}_{0, j, \downarrow}^{\dagger},
$$

where $\quad \tilde{c}_{\mathrm{k}, j, \sigma}^{\dagger}=\frac{1}{2} \sum_{\alpha} e^{i \mathrm{k} \alpha} c_{\alpha, j, \sigma}^{\dagger}$ are Fourier-transformed fermionic creation operators for a single diamond in the unit cell $j$, and $\alpha$ refers to the site labels in Fig. 1(a). The operators $\tilde{c}_{\pi, j, \sigma}^{\dagger}$ and $\tilde{c}_{0, j, \sigma}^{\dagger}$ both independently transform as the totally symmetric representation $A_{g}$ of the point group. That is to say, they commute with all the (representation) matrices $U_{g}\left(g \in D_{2 h}\right)$ for an individual diamond. As a result, $O_{\mathrm{AI}, j}^{\dagger}$ transforms according to the direct product $A_{g} \otimes A_{g} \otimes A_{g} \otimes A_{g}$, which finally turns out to be a totally symmetric representation defined in the space of four-particle states of the diamond.

Similarly, in the OAL phase at the limit $t_{1}=U=0$ and $t_{3} \gg t_{2}$, the ground state is defined by

$$
O_{\mathrm{OAL}, j}^{\dagger}=\prod_{\sigma} \frac{1}{2}\left(c_{0, j, \sigma}^{\dagger}+c_{2, j, \sigma}^{\dagger}\right)\left(c_{3, j, \sigma}^{\dagger}+c_{1, j+1, \sigma}^{\dagger}\right) .
$$

It follows that the corresponding ground state $\left|\Psi_{0}\right\rangle$ transforms trivially under all symmetries in Pmmm.

For the MI phase, considering $t_{3}=0, t_{2} / t_{1} \ll 1$ and small finite $U>0$, the ground state of the chain is described by 
Eq. (4), and the ground state of the diamond is given by

$$
O_{\mathrm{MI}, j}^{\dagger}=\frac{1}{\sqrt{2}}\left(\tilde{c}_{\frac{\pi}{2}, j, \uparrow}^{\dagger} \tilde{c}_{\frac{\pi}{2}, j, \downarrow}^{\dagger}-\tilde{c}_{-\frac{\pi}{2}, j, \uparrow}^{\dagger} \tilde{c}_{-\frac{\pi}{2}, j, \downarrow}^{\dagger}\right) \tilde{c}_{0, j, \uparrow}^{\dagger} \tilde{c}_{0, j, \downarrow}^{\dagger},
$$

which is not a single Slater determinant, and it transforms instead as $B_{1 g}$. It is odd with respect to $180^{\circ}$ rotation about the $x$ - and $y$-axis (denoted $\hat{C}_{2 x}$ and $\hat{C}_{2 y}$ ), as well as mirroring in the $y z$ - and $x z$-planes (denoted $\hat{M}_{x}$ and $\hat{M}_{y}$ ). These symmetries act on the operators of the chain as

$$
\begin{aligned}
& \hat{C}_{2 x}: c_{\alpha, j, \sigma}^{\dagger} \longmapsto i c_{\beta, j,-\sigma}^{\dagger}[A]_{\beta \alpha}, \\
& \hat{C}_{2 y}: c_{\alpha, j, \sigma}^{\dagger} \longmapsto-\sigma c_{\beta, N-j+1,-\sigma}^{\dagger}[B]_{\beta \alpha}, \\
& \hat{M}_{x}: c_{\alpha, j, \sigma}^{\dagger} \longmapsto i c_{\beta, N-j+1,-\sigma}^{\dagger}[B]_{\beta \alpha}, \\
& \hat{M}_{y}: c_{\alpha, j, \sigma}^{\dagger} \longmapsto-\sigma c_{\beta, j,-\sigma}^{\dagger}[A]_{\beta \alpha},
\end{aligned}
$$

where $\alpha, \beta$ label sites within each diamond according to Fig. 1, and $N$ is the number of diamonds in the chain. The matrices $A, B$ are given by

$$
\begin{aligned}
A & =\left(\begin{array}{llll}
0 & 0 & 1 & 0 \\
0 & 1 & 0 & 0 \\
1 & 0 & 0 & 0 \\
0 & 0 & 0 & 1
\end{array}\right), \\
B & =\left(\begin{array}{llll}
1 & 0 & 0 & 0 \\
0 & 0 & 0 & 1 \\
0 & 0 & 1 & 0 \\
0 & 1 & 0 & 0
\end{array}\right) .
\end{aligned}
$$

Since each diamond is odd with respect to these transformations, the MI ground state is an eigenstate of each operator with eigenvalue $(-1)^{N}$. As defined, when $N$ is odd, the Mott phase is distinguished from the AI and OAL because its ground state is odd under all four symmetries.

Therefore, when $N$ is odd, the MI phase can be distinguished from the trivial (AI) and obstructed (OAL) phases by, e.g., the mirror reflection eigenvalue of its ground state: the observable $\left\langle\hat{M}_{x}\right\rangle_{\Psi_{0}}=\left\langle\Psi_{0}\left|\hat{M}_{x}\right| \Psi_{0}\right\rangle$. Since odd $N$ implies one diamond being a mirror center, this is simply due to the fact that the operator $O_{\mathrm{MI}}^{\dagger}$ is odd under mirror reflection. On the other hand, when $N$ is even, the ground-state reflection eigenvalue cannot detect the MI phase. A more drastic limitation of $\left\langle\hat{M}_{x}\right\rangle_{\Psi_{0}}$ is that it cannot differentiate between the AI and the OAL phase (the same is true for $\left\langle\hat{M}_{y}\right\rangle_{\Psi_{0}}$ ).

To distinguish the AI and OAL phases, it has been proposed in Refs. [45,53,54] that the ground-state eigenvalues of partial mirror reflection operations may serve as a useful diagnostic of interacting crystalline topological phases with mirror symmetry (see Appendix $\mathrm{C}$ for details on the case of the SSH model). Many-body topological invariants for more general point-group symmetries with a combination of internal and/or Altland Zirnbauer symmetries have also been studied within a similar framework. Here, we denote a partial reflection operation twisted by $U(1)$ symmetry as $\hat{M}_{x ; I}(\theta)$. Such an operator has a nontrivial action on a restricted interval $I$ which contains the sites from $j=1$ to $j=L$ and average total $U(1)$ charge $Q_{I}$. It acts as

$$
\hat{M}_{x ; I}(\theta): c_{\alpha, j, \sigma}^{\dagger} \longmapsto i e^{-i \theta} c_{\beta, L-j+1,-\sigma}^{\dagger}[B]_{\beta \alpha}
$$

TABLE I. Expectation values of the reflection operator and $U(1)$-twisted partial reflection operator evaluated for the limiting cases defined by Eq. (4), together with Eqs. (6), (8), and (7) for the three gapped phases of the Hubbard diamond chain model: the atomic insulator (AI), the obstructed atomic limit (OAL), and the Mott insulator (MI). $Q_{I}$ denotes the average total charge enclosed within the interval $I$.

\begin{tabular}{lcc}
\hline \hline Phase & $\left\langle\hat{M}_{x}\right\rangle_{\Psi_{0}}$ & $\left\langle\hat{M}_{x ; I}(\theta)\right\rangle_{\Psi_{0}}$ \\
\hline AI & 1 & $\exp \left\{-i Q_{I}\left(\theta-\frac{\pi}{2}\right)\right\}$ \\
OAL & 1 & $\frac{1}{4} \exp \left\{-i Q_{I}\left(\theta-\frac{\pi}{2}\right)\right\} \cos ^{2} \theta$ \\
MI & $(-1)^{N}$ & $\exp \left\{-i Q_{I}\left(\theta-\frac{\pi}{2}\right)\right\}(-1)^{L}$ \\
\hline \hline
\end{tabular}

for $j \in I$ and trivially otherwise. We note that the partial symmetry operator $\hat{M}_{x ; I}(\theta)$ generally does not commute with the Hamiltonian, so the expectation value $\left\langle\hat{M}_{x ; I}(\theta)\right\rangle_{\Psi_{0}}$ may evolve continuously within a given phase. Nonetheless, it is instructive to consider the limiting cases defined by Eq. (4), together with Eqs. (6), (7), and (8). The results are summarized in Table I (see Appendix C for details).

As can be seen from Table I, the partial mirror reflection operator provides a sharper diagnostic to detect and distinguish all three phases. The factor of $e^{-i Q_{I} \theta}$ is common to all the phases and simply detects the $U(1)$ charge $Q_{I}$ enclosed within the interval $I$. The three phases can in particular be distinguished by choosing $\theta=\pi / 2$, and $L$ is an odd integer. For this choice and small $U$, the limiting values of the topological indicator are 1,0 , and -1 (since $Q_{I}$ is a multiple of 4 ) for the AI, OAL, and MI phase, respectively. If the same hoppings are considered with large $U$, these become $1, \frac{1}{2}$, and -1 , instead. Away from these ideal limits, the topological indicators are expected to remain close to the ideal values provided the correlation length remains short $[45,53,54]$, allowing them to function as a diagnostic of the ground-state topology.

The MI phase in the HDC chain is not adiabatically connected to any noninteracting atomic limit, yet it can be continued to a state with no entanglement between the unit cells (the limit $t_{3} \rightarrow 0$ ). A similar case was illuminated in Refs. [30,31]. The Hubbard diamond thus enriches the possible building blocks of quantum matter. This observation necessitates the expansion of possible atomic limits to include Mott or interacting atomic limits.

\section{TOPOLOGY AND GREEN'S FUNCTIONS}

We explore now to which extent the topology of the interacting phases in the HDC model can be identified by using eigenstate representations of the single-particle Green's function inspired by TQC. For that we make use of the concept of a topological Hamiltonian and apply CPT to calculate the Green's functions for the interacting system.

\section{A. Topological Hamiltonian}

We first briefly review the concept of the topological Hamiltonian, which allows us to define topological invariants in terms of the single-particle Green's function in an interacting system [32-34,55-60]. 
In Ref. [33] it was realized that it is sufficient to focus on the Green's function at zero frequency to obtain topological invariants. Equivalently, it is possible to define an auxiliary noninteracting Hamiltonian, referred to as a topological Hamiltonian, from which the Green's function invariants can be calculated,

$$
H_{\mathrm{T}}(\mathbf{k})=-G^{-1}(0, \mathbf{k}) .
$$

The topological Hamiltonian $H_{\mathrm{T}}$ is Hermitian and it is welldefined as long as there is a gap in the spectral function around zero frequency and $G(0, \mathbf{k})$ does not have a zero eigenvalue. Furthermore, the topological Hamiltonian possesses the same spatial symmetries as the interacting many-body Hamiltonian under the assumption that the many-body ground state is unique.

Under these conditions, it is possible to generalize the formalism of TQC and symmetry-based indicators to study the Green's functions in terms of the topological Hamiltonian [61]. Symmetry representations of valence bands and symmetry indicators can directly be computed from the topological Hamiltonian, and they can only change if either (i) the gap closes in the spectral function at zero frequency, (ii) the Green's function has a zero eigenvalue at zero frequency, or (iii) the Green's function breaks a protecting symmetry.

In what follows, we apply this formalism to our testbed HDC, where the Green's functions are obtained from CPT. The application of the formalism to a single diamond is presented in Appendix E. With the analysis below, we can gain insights into the correspondence between the topological characterization of the ground state for the interacting system presented in Sec. IV and the topological characterization performed in terms of Green's functions.

We emphasize that there is a difference between investigating the adiabatic connectedness of the ground state of an insulator and topological invariants defined in terms of the Green's function when interactions are present (see also the discussion in Refs. [62-64]).

\section{B. CPT and topological Hamiltonian for HDC}

Whereas iDMRG and VMC are powerful methods to describe the ground state of many-body low-dimensional (iDMRG) interacting systems, as shown in Sec. IV A, calculation of Green's functions with them is not straightforward. We therefore employ here CPT to obtain the Green's function of the interacting HDC model. CPT is a numerical technique for calculating the Green's functions of strongly correlated electrons described by Hubbard models in periodic lattices [35,37]. The basic idea behind CPT is to divide the lattice into a (super)lattice of clusters. The Hubbard model on each cluster is solved exactly, whereas hoppings between sites belonging to different clusters are treated perturbatively. Since the Hubbard diamond chain is suitable for the clustering in diamonds, we expect CPT to give accurate results.

Our choice of cluster is the four-site diamond [gray region in Fig. 1(a)]. Details of the method and calculations of the single-particle Green's function $G(\omega, \boldsymbol{k})$ [and spectral function $A(\omega, \boldsymbol{k})]$ for the HDC are given in Appendix D.

We first check the reliability of the CPT results for the interacting $\mathrm{HDC}$ at half-filling. For that we show in
Figs. 5(b) $-5(\mathrm{~d})$ the calculated charge gaps $\Delta / t_{1}$ [extracted from the spectral function $A(\omega, k)]$ at various values of interaction strength $U$ and along three different hopping paths in the phase diagram as marked in Fig. 5(a). We compare the results with the phase diagram obtained from iDMRG and VMC in Fig. 4. We identify four phases: Three insulators characterized by the presence of a charge gap in the spectral function, and a metallic phase, in agreement with iDMRG and VMC. Spectral functions calculated at representative points in each of these phases [marked with circles in Fig. 5(a)] are shown in Fig. 6.

The insulating phase at finite $U, t_{2} / t_{1}<1$, and $t_{3} / t_{1} \ll 1$ corresponds to the Mott phase where we identify the origin of the charge gap through the formation of upper and lower Hubbard bands [see Fig. 6(c)]. The remaining two insulating phases at $t_{2} / t_{1}>1$ are reminiscent of the noninteracting AI and OAL phases. The corresponding spectral functions suggest that they are correlated insulators [see Figs. 6(a) and 6(b)]. As $U$ increases, the phase transition connecting both phases is shifted towards larger values of $t_{3} / t_{1}$ [Fig. 5(b)], which is in good agreement with the iDMRG calculations shown in Fig. 4.

At $t_{2} / t_{1}<1$, and moderate values of $U$, a metallic phase appears between the Mott insulator and one of the correlated insulating phases in a narrow region of intermediate $t_{3} / t_{1}$ values [see Fig. 5(c)] in agreement with iDMRG and VMC. Within the range of $U$ values considered in our calculations, we observe that an increase of $U$ shifts the gap closing (opening) that indicates the onset (offset) of the metal phase to larger values of $t_{3} / t_{1}$. Since the choice of the cluster adopted for the CPT calculations treats the intercluster-hopping $t_{3}$ as a perturbation, we expect a loss in the CPT performance at large $t_{3} / t_{1}$, and therefore in that region the results are less reliable.

With the calculated Green's functions, we construct the topological Hamiltonian and proceed with the determination of the topological nature of the insulating phases appearing at $t_{2} / t_{1}>1$ by analyzing the topological Hamiltonian's spectrum. The use of the topological Hamiltonian [Eq. (16)] in this region is justified by the fact that $G(0, k)$ is nonsingular for these insulating phases. In Fig. 7, we show the spectrum of the topological Hamiltonian for both phases at $U / t_{1}=1$ together with the irreps of the little groups at $\Gamma$ and $X$ under which the topological Hamiltonian's bands transform. We note that the irreps of the occupied bands at small (large) values of $t_{3} / t_{1}$ [Figs. 7(a) and 7(b), respectively] coincide with those of the noninteracting AI (OAL) phases [compare with Figs. 3(a) and 3(c), respectively], which suggests that these interacting insulating phases are adiabatically connected to the noninteracting ones. Likewise the boundary between both AI and OAL phases is through a (charge) gap closing.

The Mott SPT phase, on the contrary, deserves special attention. In Sec. IV B it was shown that there is no smoothly connected noninteracting limit to it. A calculation of CPT Green's functions in this phase indicates that $G(0, k)$ is singular. This can be monitored by a diverging self-energy as displayed in Fig. 8 for $t_{2} / t_{1}=0.5, t_{3} / t_{1}=0.4$, and $U / t_{1}=$ 1.0. In such a situation, the topological Hamiltonian is not applicable and the EBR description cannot be pursued.

With this analysis, we conclude that the combination of the topological Hamiltonian and TQC provides a possible new 

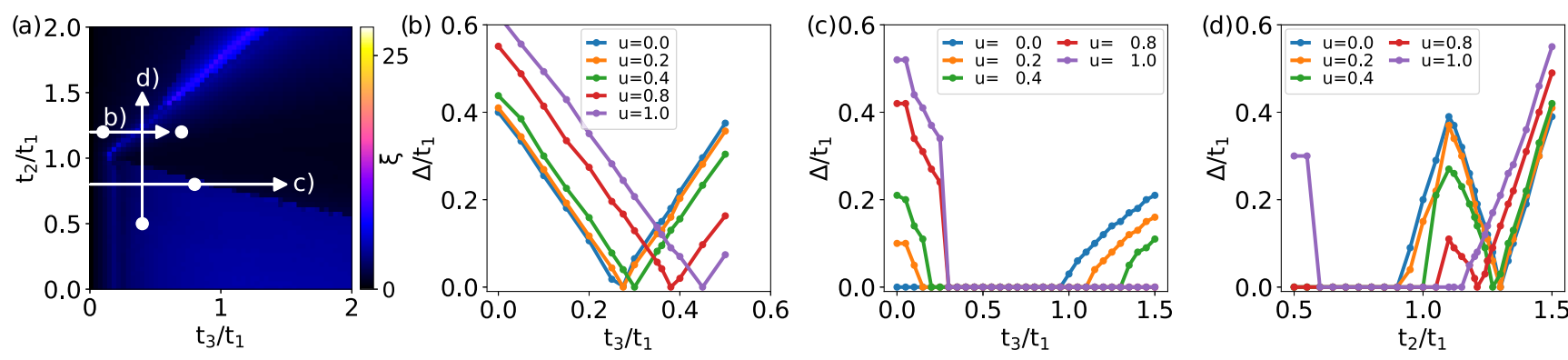

FIG. 5. (a) Labeling of the paths considered in the CPT calculations over the iDMRG/VMC phase diagram obtained with $U / t_{1}=1.0$. Parts (b), (c), and (d) show the evolution of the charge gap $\Delta / t_{1}$ along the different paths as a function of the hopping parameter for different values of the $u=U / t_{1}$. A cluster containing a single unit cell has been used in CPT calculations.

avenue to characterize correlated insulating phases as far as they are smoothly connected to noninteracting limits. This excludes Mott phases, which require a characterization beyond the single-particle Green's functions. Despite this limitation, this approach can be straightforwardly applied to diagnose the topology of many correlated insulating phases in real materials, in contrast to many-body invariants, which can be calculated only for very specific models.

\section{CONCLUSIONS AND OUTLOOK}

In this work, we have explored the possibility of extending the TQC formalism to correlated systems by studying the specific example of an extended version of a Hubbard diamond chain. After determining the phase diagram of the model using infinite density matrix renormalization group calculations and variational Monte Carlo simulations, we investigated the topology of all phases with many-body topological invariants. We thus identified three insulating phases (AI, OAL, and Mott SPT) and a metallic phase depending on the interaction strength. Specifically, we demonstrated that the Mott phase is a symmetry-protected topological phase that is not adiabati-
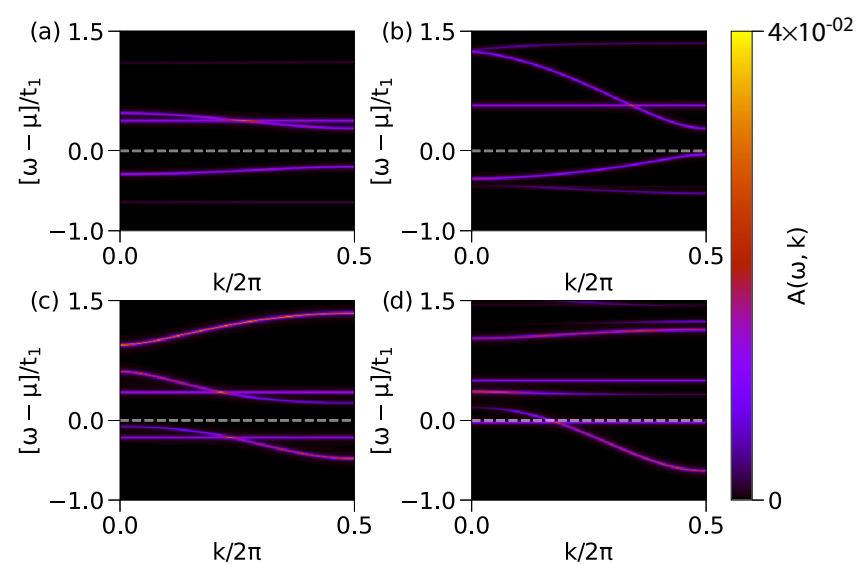

FIG. 6. CPT spectral functions $A(\omega, k)$ calculated for $U / t_{1}=$ 1 at the marked circles in Fig. 5(a). The Fermi level is shown with dashed lines. Each spectral function is normalized to satisfy $\int d \omega \sum_{k} A(\omega, k)=1$, where the integral runs over the whole frequency domain. (a) $\mathrm{AI}\left(t_{2} / t_{1}=1.2, t_{3} / t_{1}=0.1\right)$, (b) $\mathrm{OAL}\left(t_{2} / t_{1}=\right.$ $\left.1.2, t_{3} / t_{1}=0.7\right)$, (c) MI $\left(t_{2} / t_{1}=0.5, t_{3} / t_{1}=0.4\right)$, and (d) metallic phase $\left(t_{2} / t_{1}=0.8, t_{3} / t_{1}=0.8\right)$. cally connected to any band insulator, contrary to the AI and OAL phases.

Further, we investigated a generalization of the TQC formalism to Green's functions combined with the concept of topological Hamiltonians to identify the topological nature of the interacting phases, using cluster perturbation theory to calculate the Green's functions. We illustrated that this approach provides a possible recipe to characterize the topology of interacting insulating phases, as far as they are adiabatically connected to noninteracting phases. While there are many interacting phases fulfilling this condition, it excludes the detection of the topology of the Mott phase. Such a phase fundamentally requires the knowledge of $n$-particle Green's functions $(n>1)$. A systematic extension of the TQC formalism to this case may be able to identify to what type of Mott atomic limit a given phase corresponds. Before such a program can be carried out, however, the representation theory of $n$-particle Green's functions needs to be developed, which we leave for future work.

Here we focused on the example of the one-dimensional HDC with space group Pmmm, but the formalism we introduced can be easily extended to interacting systems and materials with other space groups and/or higher dimensions. This is advantageous with respect to many-body invariant calculations, which are often not achievable for material-specific models due to the mathematical complexity of such a framework. Some possible direct extensions of the present work to materials are the two-dimensional version of the diamond chain (the square-octagon lattice) recently discussed in the context of organic networks [65], or the Shastry-Sutherland
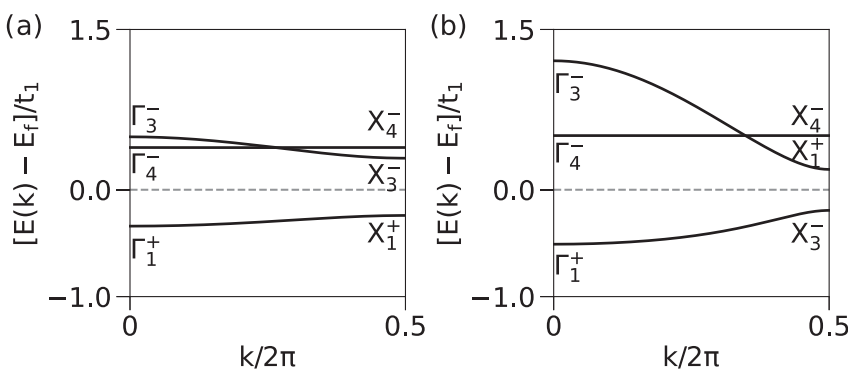

FIG. 7. Spectrum and irreps of the topological Hamiltonian in the correlated insulating phases for $U / t_{1}=1$ in (a) the AI phase $\left(t_{2} / t_{1}=1.2, t_{3} / t_{1}=0.1\right)$ and (b) the OAL phase $\left(t_{2} / t_{1}=1.2, t_{3} / t_{1}=\right.$ 0.7). The lowest occupied band has been omitted [50]. 


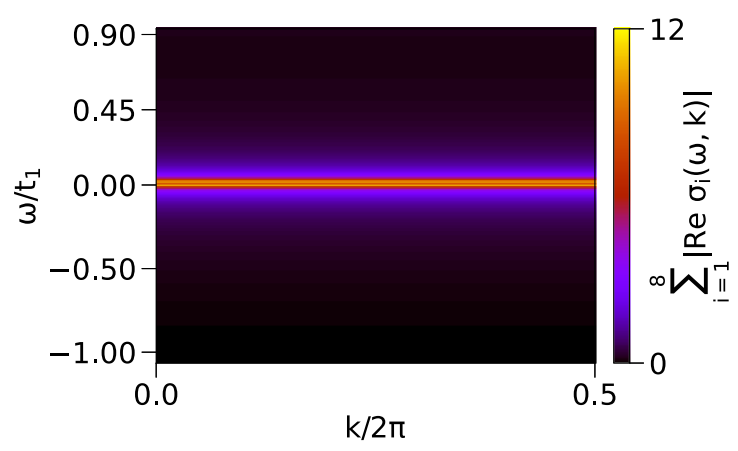

FIG. 8. Sum of the absolute value of the real part of self-energy's eigenvalues $\sigma_{i}(\omega, k)$ in the Mott SPT phase. The drastic increase close to $\omega=0$ is identified as a divergence of the self-energy.

lattice materials, which include insulating and metallic families [66], to mention a few. Applying the formalism to materials in two and three dimensions requires calculating the Green's function and irreps of the topological Hamiltonian in four and eight high-symmetry points in the BZ, respectively, and to work with space groups that contain more symmetry operations and therefore are richer in EBRs. Since irreps of little groups in high-symmetry points and EBRs of all space groups are already available [67-69], the extension of the formalism we are proposing to real materials is of practical use and may lead to new insights in the quest for diagnosing topology in interacting systems.

\section{ACKNOWLEDGMENTS}

We thank J. L. Mañes, B. A. Bernevig, B. Bradlyn, J. Cano, and F. Becca for fruitful discussions. M.G.V. and M.I. acknowledge support from the Spanish Ministerio de Ciencia e Innovacion (Grants No. PID2019-109905GB-C21 and No. PGC2018-094626-B-C21) and Basque Government (Grant No. IT979-16). F.F. acknowledges support from the Alexander von Humboldt Foundation through a postdoctoral Humboldt fellowship. N.H. acknowledges support from the Stiftung Polytechnische Gesellschaft (SPTG, Foundation Polytechnical Society Frankfurt, Germany) through a Master's fellowship. D.L., T.M., and R.V. acknowledge the Deutsche Forschungsgemeinschaft (DFG, German Research Foundation) for funding through Grant No. TRR 288422213477 (project B05). A.T. acknowledges funding by the European Union's Horizon 2020 research and innovation program under the Marie Sklodowska Curie grant agreement No. 701647. Part of the work of M.G.V., F.P., and R.V. was carried out at Kavli Institute of Theoretical Physics (KITP), which is supported by the National Science Foundation under Grant No. NSF PHY-1748958. T.N. and F.P. acknowledges funding from the European Union's Horizon 2020 research and innovation program (ERC-StG-Neupert-757867-PARATOP and ERC-CoG-Pollmann-771537-DYNACQM).

\section{APPENDIX A: DMRG CALCULATIONS}

The density matrix renormalization group (DMRG) algorithm is one of the most powerful and unbiased numerical methods for one-dimensional and quasi-one-dimensional systems [70]. Our calculations have been performed using the (a)
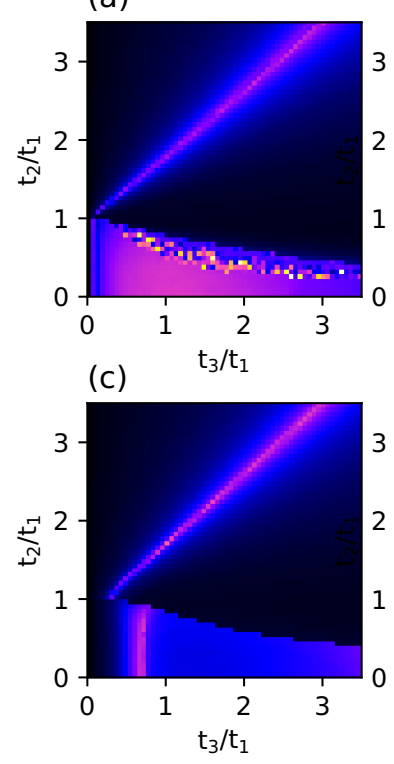

(b)
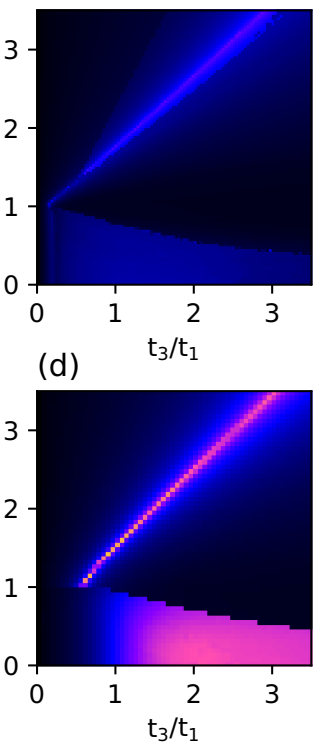

25

$-20$

$-15$ $\xi$
FIG. 9. iDMRG calculations for the HDC model showing the correlation length $\xi$ for Hubbard interaction strengths (a) $U / t_{1}=0.4$, (b) $U / t_{1}=1.0$, (c) $U / t_{1}=2.0$, and (d) $U / t_{1}=4.0$. The maximal bond dimension is set to $\chi=128$.

infinite DMRG (iDMRG) method [39], which is an extension of standard DMRG to infinite systems, as implemented in the TENPY package [71].

We initialize the algorithm on a two-diamond unit cell as the half-filled product state $|\Psi\rangle_{0}=|\downarrow, \downarrow, \uparrow, \uparrow, \downarrow, \downarrow, \uparrow, \uparrow\rangle$, with the sites ordered as given in Fig. 1. From there, we build a matrix product state (MPS) representation of the form

$$
|\Psi\rangle=\sum_{j_{1} \ldots j_{N}} M^{[1] j_{1}} M^{[2] j_{2}} \cdots M^{[N] j_{N}}\left|j_{1}, j_{2}, \ldots, j_{N}\right\rangle,
$$

where each $M^{[n] j_{n}}$ is a $\chi_{n} \times \chi_{n+1}$ matrix, and $N$ is the number of sites. We employ the commonly used two-site update, which sweeps through the system and iteratively optimizes the matrices by minimizing the energy locally with respect to our Hamiltonian (1), keeping the number of electrons fixed. The procedure is repeated until the convergence criteria are fulfilled $\left(\Delta E_{\text {rel }}<10^{-10}\right.$ and $\left.\Delta S<10^{-4}\right)$.

Having calculated the ground state, we compute the correlation length, which, in the DMRG context, is defined as

$$
\xi=-\frac{N}{\ln \left|\eta_{2}\right|}
$$

with $\eta_{2}$ denoting the second largest eigenvalue of the transfer matrix $T$ [71]. While gapped phases are characterized by a finite correlation length, critical points, as well as metallic phases, have a diverging $\xi$ [52]. The resulting phase diagrams are presented in Fig. 9. The noninteracting phase diagram as shown in Fig. 2 is certainly recognizable in the DMRG results.

Although formally the correlation length $\xi$ diverges at the phase boundaries, it only assumes a large finite value in our data, since it is bounded by the maximal bond dimension, which is set to $\chi=128$. For metallic or close-to-metallic systems, DMRG generally performs poorly, resulting in points 


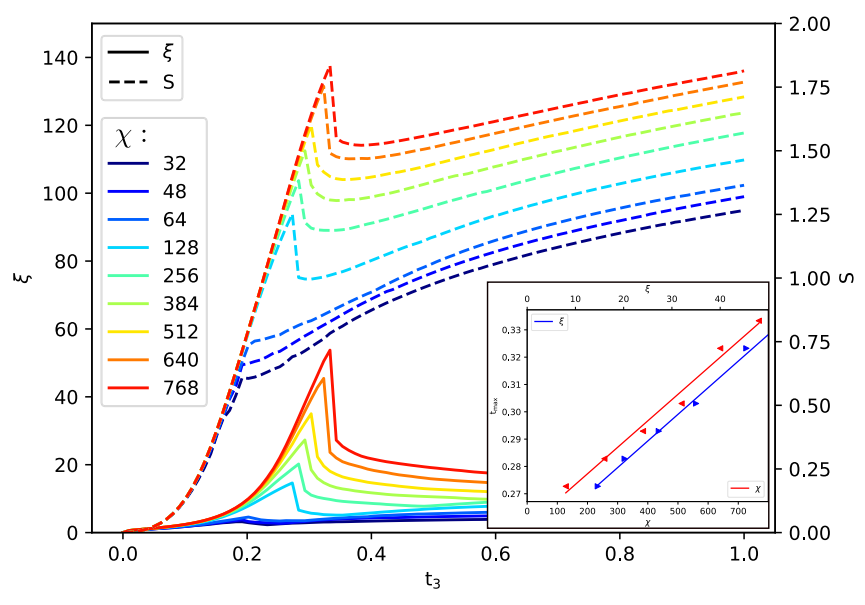

FIG. 10. Correlation length $\xi$ (solid line) and entanglement entropy $S$ (dashed line) plotted against $t_{3} / t_{1}$ for $U / t_{1}=1.0$ and $t_{2} / t_{1}=$ 0.3 for different values of $\chi$.

that are not fully converged close the lower phase boundary for $U=0.4$ in Fig. 9(a).

For $t_{2}=0$ and $t_{3} \ll t_{1}$, we expect a Mott-insulating phase for any finite value of $U$. Increasing the Hubbard interaction, the short-range correlated Mott region in the lower left-hand corner of the phase diagram extends further to the right. Increasing $t_{3}$, the system either undergoes a transition into an intermediate metallic phase, or, for sufficiently large values of $U$, it enters the OAL phase directly. The exact value of $U$ at which the intermediate phase is completely suppressed is hard to pinpoint, due to the strong drift observed in the data.

Fixing $t_{2} / t_{1}=0.3$ and $U / t_{1}=1.0$, we plot the correlation length $\xi$ and the entanglement entropy $S$ against $t_{3} / t_{1}$ for different maximal bond dimensions $\chi$ in Fig. 10. With increasing $\chi$, we note that the position of the peak $t_{\max }$ shifts to higher values of $t_{3} / t_{1}$ as shown in the inset of the figure. Extrapolating this behavior to infinitely large values of $\chi$ suggests that the Mott-metal transition is suppressed, and the system enters the OAL phase directly.

Using finite DMRG, we calculate the charge gap crossing the AI-OAL phase boundary at $t_{2} / t_{1}=1.2$ with $U / t_{1}=1.0$ on a chain of 20 diamonds ( 80 sites). As in the noninteracting case, we observe a closure of the charge gap at the transition.

\section{APPENDIX B: VARIATIONAL MONTE CARLO CALCULATIONS}

To strengthen our results for the phase diagram of the diamond chain, we also perform variational Monte Carlo (VMC) calculations in the region of the phase diagram in which the metallic phase is observed $\left(t_{2} / t_{1}<1\right)$. Our variational approach is based on Jastrow-Slater wave functions of the form

$$
\left|\Psi_{\mathrm{var}}\right\rangle=\mathcal{J}_{n} \mathcal{J}_{s}\left|\Phi_{0}\right\rangle,
$$

in which long-range Jastrow correlators, $\mathcal{J}_{n}$ and $\mathcal{J}_{s}$, are applied onto an uncorrelated fermionic state, $\left|\Phi_{0}\right\rangle$, to introduce nontrivial electron-electron correlations. This class of variational states has been shown to accurately describe both metallic and Mott insulating phases in one dimension [42]. The variational Ansatz of Eq. (B1) features long-range density-density and spin-spin Jastrow factors,

$$
\begin{aligned}
& \mathcal{J}_{n}=\exp \left(\sum_{i, j} \sum_{\alpha, \beta} v_{\alpha, i ; \beta, j} n_{\alpha, i} n_{\beta, j}\right), \\
& \mathcal{J}_{s}=\exp \left(\sum_{i, j} \sum_{\alpha, \beta} u_{\alpha, i ; \beta, j} S_{\alpha, i}^{z} S_{\beta, j}^{z}\right),
\end{aligned}
$$

and the noninteracting state $\left|\Phi_{0}\right\rangle$. Although the simplest choice for $\left|\Phi_{0}\right\rangle$ is the ground state of the Hamiltonian (1) with $U=0$, here we adopt a more general scheme, in which we consider the ground state of an auxiliary quadratic Hamiltonian [41],

$$
\begin{aligned}
\mathcal{H}_{0}= & \sum_{i, j} \sum_{\alpha, \beta}\left[\sum_{\sigma} t_{\alpha, i ; \beta, j} c_{\alpha, i, \sigma}^{\dagger} c_{\beta, j, \sigma}+\right.\text { H.c. } \\
& \left.+\Delta_{\alpha, i ; \beta, j}\left(c_{\alpha, i, \uparrow}^{\dagger} c_{\beta, j, \downarrow}^{\dagger}+c_{\beta, j, \uparrow}^{\dagger} c_{\alpha, i, \downarrow}^{\dagger}\right)+\text { H.c. }\right] \\
& +\Delta_{\mathrm{AF}} \sum_{j} \sum_{\alpha}\left[e^{i \pi(j+\alpha)} c_{\alpha, j, \uparrow}^{\dagger} c_{\alpha, j, \downarrow}+\text { H.c. }\right] .
\end{aligned}
$$

$\mathcal{H}_{0}$ contains hopping terms $\left(t_{\alpha, i ; \beta, j}\right)$ and singlet pairing terms $\left(\Delta_{\alpha, i ; \beta, j}\right)$ up to fifth neighbors, and a Néel magnetic field $\left(\Delta_{\mathrm{AF}}\right)$. To minimize the variational energy of the trial state, all the parameters of $\mathcal{H}_{0}$ and the Jastrow pseudopotentials $\left(v_{\alpha, i ; \beta, j}, u_{\alpha, i ; \beta, j}\right)$ are optimized by means of the stochastic reconfiguration technique [41,72].

When scanning the phase diagram of the diamond chain, we can discriminate between metallic and insulating phases by computing two distinct observables. On the one hand, we can evaluate the density-density structure factor $N(q)=$ $\left\langle n_{-q} n_{q}\right\rangle_{\mathrm{var}}$, where $n_{q}=N^{-1} \sum_{j, \alpha} n_{\alpha, j} \exp (i q j)$ is the Fourier transform of the density operator, and $\langle\cdots\rangle_{\text {var }}$ indicates the expectation value with respect to the variational state (B1). The absence (presence) of a charge gap is signaled by the linear (quadratic) behavior of $N(q)$ for $q \rightarrow 0[42,43,73]$. On the other hand, we can compute the expectation value of the localization parameter introduced in Ref. [74], namely

$$
z_{L}=\left\langle\exp \left(\frac{2 \pi i}{N} \sum_{j, \alpha} j n_{\alpha, j}\right)\right\rangle_{\mathrm{var}} .
$$

In the thermodynamic limit, $\left|z_{L}\right| \rightarrow 0$ in a metallic phase while $\left|z_{L}\right| \rightarrow 1$ in an insulating phase [42] (see Fig. 11 for an example).

We performed VMC calculations for $U / t_{1}=0.4$ and 1 , $t_{2} / t_{1}=0.5$ and 0.8 , and different values of $t_{3} / t_{1}$. The results are reported in Fig. 4 on top of the DMRG phase diagram.

\section{APPENDIX C: BENCHMARKING THE TOPOLOGICAL INVARIANTS WITH THE SU-SCHRIEFFER-HEEGER MODEL}

Let us consider the fixed point Su-Schrieffer-Heeger (SSH) model described by the Hamiltonian

$$
H(\alpha)=\sum_{j=1}^{N-1} b_{j}^{\dagger} a_{j+1}+e^{-i \alpha} b_{N}^{\dagger} a_{1}+\text { H.c. },
$$



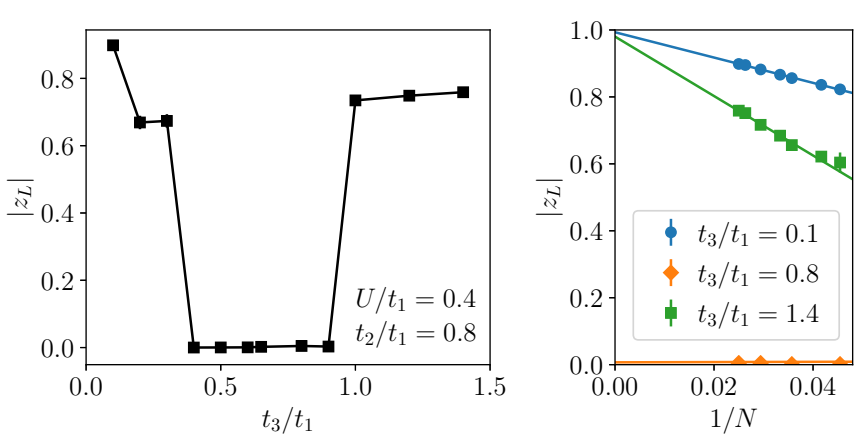

FIG. 11. Localization parameter $z_{L}$ [Eq. (B5)] computed by variational Monte Carlo for $U / t_{1}=0.4$ and $t_{2} / t_{1}=0.8$. Left panel: $\left|z_{L}\right|$ for a system of $N=40$ diamonds (160 sites) and different values of $t_{3} / t_{1}$. We observe a metallic phase $\left(\left|z_{L}\right| \approx 0\right)$ sandwiched between two insulating phases (finite $\left|z_{L}\right|$ ). Right panel: Finite-size scaling of $\left|z_{L}\right|$ at $t_{3} / t_{1}=0.1$ (insulator), 0.8 (metal), 1.4 (insulator).

where we have inserted a $U(1)$ flux by twisting the boundary conditions by $e^{-i \alpha}$. Let us define basis transformed fermions as

$$
\begin{aligned}
& f_{j+\frac{1}{2}}^{\dagger}=\frac{1}{\sqrt{2}}\left(b_{j}^{\dagger}-a_{j+1}^{\dagger}\right), \\
& \widetilde{f}_{j+\frac{1}{2}}^{\dagger}=\frac{1}{\sqrt{2}}\left(b_{j}^{\dagger}+a_{j+1}^{\dagger}\right)
\end{aligned}
$$

for $j=1, \ldots, N-1$ and

$$
\begin{aligned}
& f_{\frac{1}{2}}^{\dagger}(\alpha)=\frac{1}{\sqrt{2}}\left(b_{N}^{\dagger}-e^{-i \alpha} a_{1}^{\dagger}\right), \\
& \tilde{f}_{\frac{1}{2}}^{\dagger}(\alpha)=\frac{1}{\sqrt{2}}\left(b_{N}^{\dagger}+e^{-i \alpha} a_{1}^{\dagger}\right) .
\end{aligned}
$$

Then the ground state takes the form

$$
|\Psi(\alpha)\rangle_{\mathrm{SSH}}=\prod_{j} f_{j+\frac{1}{2}}^{\dagger}|0\rangle,
$$

where $\prod_{j} f_{j+1 / 2}^{\dagger}=f_{1 / 2}^{\dagger}(\alpha) f_{3 / 2}^{\dagger} \cdots f_{N-1 / 2}^{\dagger}$. First we compute the ground-state eigenvalue for the mirror operator $\hat{M}_{x}$, which has the following action:

$$
\hat{M}_{x}:\left[\begin{array}{l}
a^{\dagger} \\
b^{\dagger}
\end{array}\right]_{j} \mapsto\left[\begin{array}{l}
b^{\dagger} \\
a^{\dagger}
\end{array}\right]_{N-j+1},
$$

therefore it can be immediately read off that

$$
\hat{M}_{x}:\left[\begin{array}{c}
f_{j+\frac{1}{2}}^{\dagger} \\
f_{\frac{1}{2}}^{\dagger}(\alpha)
\end{array}\right] \mapsto\left[\begin{array}{c}
-f_{N-j+\frac{1}{2}}^{\dagger} \\
-e^{-i \alpha} f_{\frac{1}{2}}^{\dagger}(-\alpha)
\end{array}\right],
$$

with which one can explicitly show that

$$
{ }_{\operatorname{SSH}}\left\langle\Psi(\alpha)\left|\hat{M}_{x}\right| \Psi(\alpha)\right\rangle_{\mathrm{SSH}}=(-1)^{\frac{N}{2}(N-1)+1} \cos (\alpha) .
$$

This quantity by itself does not carry topological information. Furthermore, when $\alpha=0$ the quantity still depends on $N$ itself and not just its parity. Instead, as shown in Ref. [53], one may define a many-body invariant as

$$
\begin{aligned}
\gamma_{\mathrm{SSH}} & :=e^{\oint \mathrm{d} \alpha_{\mathrm{SSH}}\left\langle\Psi(\alpha)\left|\partial_{\alpha}\right| \Psi(\alpha)\right\rangle_{\mathrm{SSH}}} \\
& =\frac{\operatorname{SSH}\left\langle\Psi(\pi)\left|\hat{M}_{x}\right| \Psi(\pi)\right\rangle_{\mathrm{SSH}}}{\operatorname{SSH}_{\mathrm{SS}}\left\langle\Psi(0)\left|\hat{M}_{x}\right| \Psi(0)\right\rangle_{\mathrm{SSH}}}=-1 .
\end{aligned}
$$

Further, it is known that the interaction classification of class A insulators with additional mirror reflection symmetry with $M_{x}^{2}=+1$ is given by the cobordism group $\Omega_{\text {pin }}^{2}$ (pt.) $=\mathbb{Z}_{4}$. Therefore, the above many-body invariant is not capable of detecting such a classification. To capture the refined interacting classification, the partial reflection operation may be used. We consider the $U(1)$-twisted partial mirror reflection operator $\hat{M}_{x, I}(\theta)$ which acts on the interval $I$ containing sites $j=1$ to $j=L$. The operator acts as

$$
\hat{M}_{x, I}(\theta):\left[\begin{array}{l}
a^{\dagger} \\
b^{\dagger}
\end{array}\right]_{j} \mapsto e^{-i \theta}\left[\begin{array}{l}
b^{\dagger} \\
a^{\dagger}
\end{array}\right]_{L-j+1}
$$

while the action of $\hat{M}_{x, I}(\theta)$ in the bond basis takes the form

$$
\begin{aligned}
\hat{M}_{\theta, I}: f_{\frac{1}{2}}^{\dagger} & \mapsto\left(b_{N}^{\dagger}-e^{-i \theta} b_{L}^{\dagger}\right) / \sqrt{2} f_{L+\frac{1}{2}}^{\dagger} \\
& \mapsto\left(e^{-i \theta} a_{1}^{\dagger}-a_{L+1}^{\dagger}\right) / \sqrt{2} f_{j+\frac{1}{2}}^{\dagger} \mapsto-e^{-i \theta} f_{L-j+\frac{1}{2}}
\end{aligned}
$$

for $j \in[1, \ldots, L-1]$. For all other operators, the partial reflection acts trivially. The partial reflection eigenvalue can be computed as

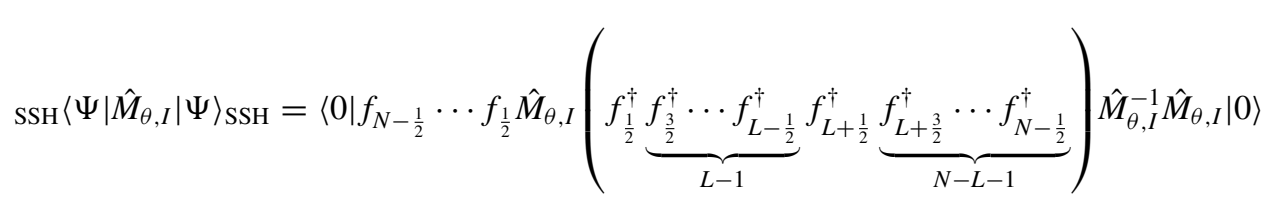

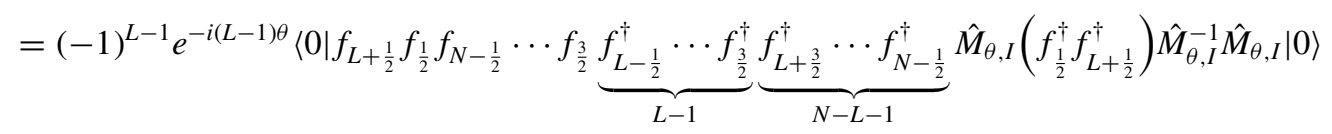

$$
\begin{aligned}
& =(-1)^{L-1+\sum_{n=1}^{L-2} 1} e^{-i(L-1) \theta}\left\langle 0\left|f_{L+\frac{1}{2}} f_{\frac{1}{2}} \hat{M}_{\theta, I}\left(f_{\frac{1}{2}}^{\dagger} f_{L+\frac{1}{2}}^{\dagger}\right) \hat{M}_{\theta, I}^{-1} \hat{M}_{\theta, I}\right| 0\right\rangle \\
& =\frac{1}{4}(-1)^{\frac{L}{2}(L-1)} e^{-i(L-1) \theta}\left\langle 0\left|f_{L+\frac{1}{2}} f_{\frac{1}{2}}\left(b_{N}^{\dagger}-e^{-i \theta} b_{L}^{\dagger}\right)\left(e^{-i \theta} a_{1}^{\dagger}-a_{L+1}^{\dagger}\right)\right| 0\right\rangle \\
& =\frac{i}{2} e^{-i L \theta} \sin \theta(-1)^{L(L-1) / 2},
\end{aligned}
$$


where we have used the shorthand $M_{\theta, I}$ for $M_{x, I}(\theta)$. It can be seen that for $\theta= \pm \pi / 2$ and $L$ even, the partial reflection operation produces a phase of $\pm i$ which is a topological diagnostic of $\mathbb{Z}_{4}=\Omega_{\text {pin }}^{2}$. Conversely, if we consider odd $L$, i.e., site-centered inversion, we obtain $\arg \left(\operatorname{ssH}_{\text {SH }}\left\langle\Psi\left|\hat{M}_{\theta, 0}\right| \Psi\right\rangle_{\text {SSH }}\right) \in$ $\{0, \pi\}$, which implies a $\mathbb{Z}_{2}$ invariant.

\section{APPENDIX D: CLUSTER PERTURBATION THEORY}

We briefly introduce CPT and its implementation to obtain momentum-resolved spectral functions for generalized Hubbard models on a lattice. The basic idea behind CPT is to divide the lattice into a superlattice of clusters. The Hubbard model on each cluster is solved exactly, whereas the hoppings between sites belonging to different clusters are treated perturbatively. More details about the method and its applicability can be found in Refs. [35-38,75].

We consider the general form of the Hubbard Hamiltonian:

$$
H=\sum_{r r^{\prime} \sigma} t_{r \sigma, r^{\prime} \sigma} c_{r \sigma}^{\dagger} c_{r^{\prime} \sigma}+\sum_{r} U n_{r \uparrow} n_{r \downarrow},
$$

where $c_{r \sigma}^{\dagger}\left(c_{r \sigma}\right)$ creates (annihilates) an electron with spin $\sigma$ at site $\boldsymbol{r}$, and $t_{\boldsymbol{r} \sigma, \boldsymbol{r}^{\prime} \sigma}$ is the hopping amplitude of an electron with spin $\sigma$ from site $\boldsymbol{r}^{\prime}$ to $\boldsymbol{r}$.

The kinetic term of Eq. (D1) can be written in a form that shows the tiling of the lattice into clusters:

$$
H=\sum_{i j} \mathbf{c}_{i}^{\dagger} t^{(i, j)} \mathbf{c}_{j}+\sum_{\boldsymbol{r}} U n_{\boldsymbol{r} \uparrow} n_{\boldsymbol{r} \downarrow},
$$

where $i, j=1, \ldots, L$, with $L$ the number of clusters in the crystal. $t^{(i, j)}$ is the block of the hopping matrix containing terms coupling sites belonging to cluster $\xi_{i}$ to those of cluster $\xi_{j}$, and $\mathbf{c}_{j}$ is the column-vector of annihilation operators corresponding to sites in cluster $\xi_{j}$. The Hamiltonian $H^{(i)}$ of a particular cluster is obtained by choosing from Eq. (D2) the kinetic and interaction terms that involve only sites within the cluster $\xi_{i}$. Mathematically, this corresponds to taking a block matrix $t^{(i, i)}$ in the diagonal of the hopping matrix:

$$
H^{(i)}=\mathbf{c}_{i}^{\dagger} t^{(i, i)} \mathbf{c}_{i}+\sum_{\boldsymbol{r} \in \xi_{i}} U n_{\boldsymbol{r} \uparrow} n_{\boldsymbol{r} \downarrow} .
$$

The ground state of $H^{(i)}$ is calculated with exact diagonalization $[75,76]$ and is used to construct the cluster Green's function $G^{(i)}(\omega)$ :

$$
G^{(i)}(\omega)=\left[\omega-t^{(i, i)}-\Sigma^{(i)}(\omega)\right]^{-1},
$$

where $\Sigma^{(i)}(\omega)$ is the self-energy of $\xi_{i}$. The main approximation of CPT consists of constructing the lattice self-energy $\Sigma(\omega)$ as a direct sum of cluster self-energies, i.e., as a blockdiagonal matrix where each block is the self-energy of a cluster:

$$
\Sigma(\omega)=\bigoplus_{i} \Sigma^{(i)}(\omega) .
$$

The Dyson equation relating the lattice Green's function $G(\omega)$ and self-energy $\Sigma(\omega)$ reads

$$
[G(\omega)]^{-1}=\omega-t-\Sigma(\omega),
$$

where $t$ is the hopping matrix. Combining Eqs. (D4), (D5), and (D6) leads to the following expression for $G(\omega)$ :

$$
[G(\omega)]^{-1}=\bigoplus_{i}\left[G^{(i)}(\omega)\right]^{-1}-t_{\text {inter }} .
$$

Here, $t_{\text {inter }}$ denotes the matrix obtained by removing the blocks in the diagonal of the hopping matrix $t$, i.e., the hopping matrix including only terms that couple different clusters. Written in matrix form, Eq. (D7) reads

$$
[G(\omega)]^{-1}=\left(\begin{array}{cccc}
{\left[G^{(1)}(\omega)\right]^{-1}} & -t^{(1,2)} & \cdots & -t^{(1, L)} \\
-t^{(2,1)} & {\left[G^{(2)}(\omega)\right]^{-1}} & \cdots & -t^{(2, L)} \\
\vdots & \vdots & \ddots & \vdots \\
-t^{(L, 1)} & -t^{(L, 2)} & \cdots & {\left[G^{(L)}(\omega)\right]^{-1}}
\end{array}\right) .
$$

CPT inherits its name from the fact that Eq. (D7) can be derived by isolating $t_{\text {inter }}$ in Eq. (D2), treating it as a perturbation to the rest and conserving only first-order terms $[37,77]$.

Even if position indices have not been written explicitly, the Green's function $G(\omega)$ in Eq. (D7) is written in real space. However, in order to derive the momentum-resolved spectral function $A(\omega, \boldsymbol{k})=-\pi^{-1} \operatorname{Im} G\left(\omega+i 0^{+}, \boldsymbol{k}\right)$ and the topological Hamiltonian, it is convenient to calculate its reciprocal space representation $G(\omega, \boldsymbol{k})$ by applying a periodization formula derived below that Fourier transforms Eq. (D7) to reciprocal space.

We introduce now the concept of a supercell and work out the kinematics of a lattice tiled into clusters, which will lead us to the periodization formula relating the $\boldsymbol{k}$-resolved Green's function to the real-space Green's function calculated by CPT.

A supercell is defined as a unit cell containing a group of clusters. When all clusters are of the same kind, i.e., when all $H^{(i)}$ are related by a translation of $\gamma$, a supercell containing a single cluster may be chosen [see Figs. 12(a) and 12(b)]. Generally, the smallest possible supercell may contain many clusters [see Fig. 12(c)]. Note that supercells form a superlattice $\Gamma$, which is part of the original lattice $\gamma$, so that $\Gamma \subset \gamma$.

Let us consider an atom of the crystal. We denote by $\boldsymbol{r}$ the position of the unit cell it belongs to, while the corresponding supercell and unit cell within the supercell are indicated by $\tilde{\boldsymbol{r}}$ and $\boldsymbol{R}$, respectively; thus, we can write $\boldsymbol{r}=\tilde{\boldsymbol{r}}+\boldsymbol{R}$ (see Fig. 13). In reciprocal space, any vector $\boldsymbol{k}$ in the Brillouin zone of $\gamma$ can be written as $\boldsymbol{k}=\tilde{\boldsymbol{k}}+\boldsymbol{K}$, where $\tilde{\boldsymbol{k}}$ belongs to the Brillouin zone of $\Gamma$, and $\boldsymbol{K}$ belongs to the reduced reciprocal lattice corresponding to $\Gamma$. One-body functions expressed in terms of $\boldsymbol{k}, \tilde{\boldsymbol{k}}$, and $\boldsymbol{K}$ are related, via the following Fourier transforms, to the descriptions that depend on $\boldsymbol{r}, \tilde{\boldsymbol{r}}$, and $\boldsymbol{R}$ :

$$
\begin{aligned}
f(\boldsymbol{k}) & =f(\tilde{\boldsymbol{k}}+\boldsymbol{K})=\frac{1}{\sqrt{N}} \sum_{\boldsymbol{r}} e^{-i \boldsymbol{k} \cdot \boldsymbol{r}} f(\boldsymbol{r}), \\
f(\tilde{\boldsymbol{k}}) & =\frac{1}{\sqrt{N_{\Gamma}}} \sum_{\tilde{\boldsymbol{r}}} e^{-i \tilde{\boldsymbol{k}} \cdot \tilde{\boldsymbol{r}}} f(\tilde{\boldsymbol{r}}), \\
f(\boldsymbol{K}) & =\frac{\sqrt{N_{\Gamma}}}{\sqrt{N}} \sum_{\boldsymbol{R}} e^{-i \boldsymbol{K} \cdot \boldsymbol{R}} f(\boldsymbol{R}),
\end{aligned}
$$

where $N_{\Gamma}$ is the number of supercells in the lattice. We can define two reciprocal space representations: On the one hand, we have the $\boldsymbol{k}$-representation, based on the transformation of Eq. (D8a). On the other hand, the ( $\tilde{\boldsymbol{k}}, \boldsymbol{K})$-representation is obtained by consecutive application of Eqs. (D8b) and 


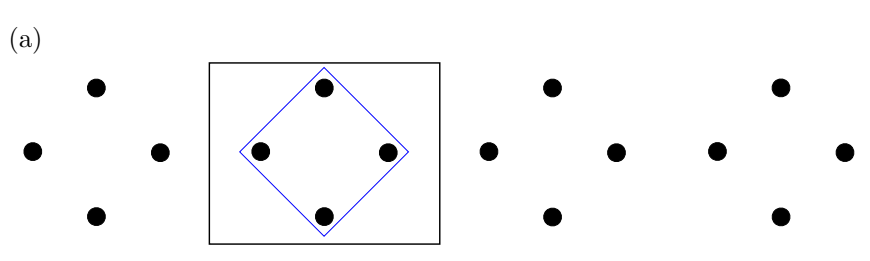

(b)

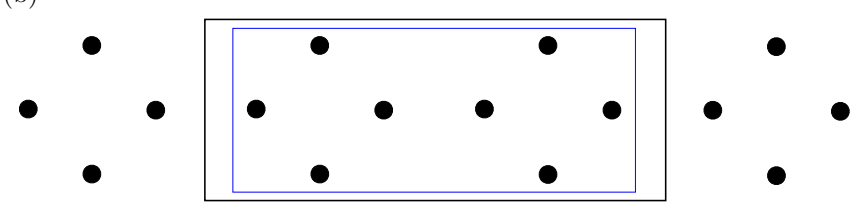

(c)

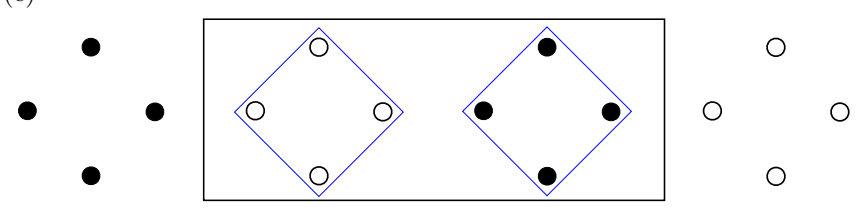

FIG. 12. Different clusters and supercells of diamondlike chains. Supercells are marked with black lines, clusters with blue lines. (a) Diamond chain, where a cluster contains a single diamond and a supercell contains a single cluster. (b) Diamond chain, with a cluster containing two diamonds and a supercell containing a single cluster. (c) Diamondlike chain, constructed by placing successively two different diamonds. Each cluster contains a single diamond, and the smallest supercell that can be chosen contains two clusters.

(D8c). Each of these representations relates the real- and reciprocal-space representations of the annihilation operators in the following form:

$$
\begin{aligned}
c(\boldsymbol{k}) & =\frac{1}{\sqrt{N}} \sum_{r} e^{-i \boldsymbol{k} \cdot \boldsymbol{r}} c(\boldsymbol{r}), \\
c_{\boldsymbol{K}}(\tilde{\boldsymbol{k}}) & =\frac{1}{\sqrt{N}} \sum_{\boldsymbol{R}} \sum_{\boldsymbol{r}} e^{-i \tilde{\boldsymbol{k}} \cdot \tilde{\boldsymbol{r}}} e^{-i \boldsymbol{K} \cdot \boldsymbol{R}} c(\tilde{\boldsymbol{r}}+\boldsymbol{R}) .
\end{aligned}
$$

Here, Eq. (D9a) corresponds to the $\boldsymbol{k}$-representation and Eq. (D9b) to the $(\tilde{\boldsymbol{k}}, \boldsymbol{K})$-representation. The matrix $\Delta$ relating

(a)

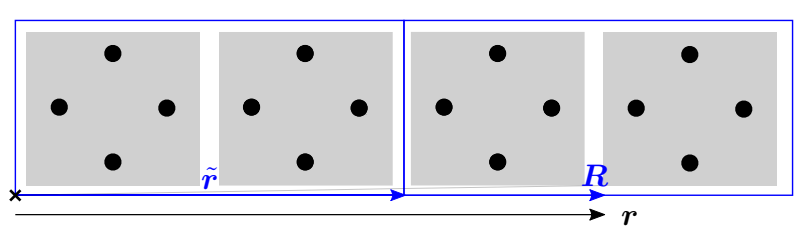

(b)

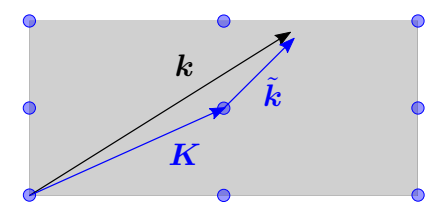

FIG. 13. Illustration of the notation. (a) Example of a possible choice of the supercell (blue) in the HDC, where the unit cell is marked in gray. (b) Reciprocal structure, with the BZ in gray and sites of the superlattice denoted by blue dots. both representations, $c(\boldsymbol{k})=\Delta_{\boldsymbol{k}, \tilde{\boldsymbol{k}}^{\prime} \boldsymbol{K}^{\prime}} c_{\boldsymbol{K}^{\prime}}\left(\tilde{\boldsymbol{k}}^{\prime}\right)$, is the following:

$$
\begin{aligned}
\Delta_{\boldsymbol{k}, \tilde{\boldsymbol{k}}^{\prime} \boldsymbol{K}^{\prime}} & =\frac{1}{N} \sum_{\tilde{\boldsymbol{r}} \boldsymbol{R}} e^{-i \boldsymbol{k} \cdot(\tilde{\boldsymbol{r}}+\boldsymbol{R})} e^{i \tilde{\boldsymbol{k}}^{\prime} \cdot \tilde{\boldsymbol{r}}} e^{i \boldsymbol{K}^{\prime} \cdot \boldsymbol{R}} \\
& =\delta_{\tilde{\boldsymbol{k}} \tilde{\boldsymbol{k}}^{\prime}} \frac{N_{\Gamma}}{N} \sum_{\boldsymbol{R}} e^{-i\left(\tilde{\boldsymbol{k}}+\boldsymbol{K}-\boldsymbol{K}^{\prime}\right) \cdot \boldsymbol{R}}
\end{aligned}
$$

where $\boldsymbol{k}=\tilde{\boldsymbol{k}}+\boldsymbol{K}$. Note that $\Delta$ is not diagonal for all $\boldsymbol{k}$ and $\left(\tilde{\boldsymbol{k}}^{\prime}, \boldsymbol{K}^{\prime}\right)$, which means that the representations are not equivalent. In addition, we can set a $(\tilde{\boldsymbol{k}}, \boldsymbol{R})$-representation, which lies midway between both $\boldsymbol{k}$ - and $(\tilde{\boldsymbol{k}}, \boldsymbol{K})$-representations:

$$
c_{\boldsymbol{R}}(\tilde{\boldsymbol{k}})=\frac{1}{\sqrt{N_{\Gamma}}} \sum_{\tilde{\boldsymbol{r}}} e^{-i \tilde{\boldsymbol{k}} \cdot \tilde{r}} c(\tilde{\boldsymbol{r}}+\boldsymbol{R}) .
$$

Generally, treating inter- and intracluster hoppings differently breaks the invariance under translations of $\gamma$, thus $G(\omega)$ in Eq. (D7) is not diagonal in the $\boldsymbol{k}$-representation. Nevertheless, since invariance under translations of $\Gamma$ is preserved, it is diagonal in $\tilde{\boldsymbol{k}}$-indices. Therefore, it is convenient to express $G(\omega)$ in the $(\tilde{\boldsymbol{k}}, \boldsymbol{R})$ representation:

$$
[G(\omega, \tilde{\boldsymbol{k}})]^{-1}=\bigoplus_{i}\left[G^{(i)}(\omega)\right]^{-1}-t_{\mathrm{inter}}(\tilde{\boldsymbol{k}}),
$$

where $\boldsymbol{R}$ and $\boldsymbol{R}^{\prime}$ indices have been omitted. To achieve the $\boldsymbol{k}$-representation of the Green's function, we first write it in the $(\tilde{\boldsymbol{k}}, \boldsymbol{K})$-representation:

$$
G_{\boldsymbol{K} \boldsymbol{K}^{\prime}}(\omega, \tilde{\boldsymbol{k}})=\frac{N_{\Gamma}}{N} \sum_{\boldsymbol{R} \boldsymbol{R}^{\prime}} e^{i \boldsymbol{K}^{\prime} \cdot \boldsymbol{R}^{\prime}} e^{-i \boldsymbol{K} \cdot \boldsymbol{R}} G_{\boldsymbol{R} \boldsymbol{R}^{\prime}}(\omega, \tilde{\boldsymbol{k}})
$$

Applying the matrix $\Delta$ of Eq. (D10) to $G_{\boldsymbol{K} K^{\prime}}(\omega, \tilde{\boldsymbol{k}})$ leads to the following expression of the Green's function in the $\boldsymbol{k}$ representation:

$$
G\left(\omega, \boldsymbol{k}, \boldsymbol{k}^{\prime}\right)=\frac{N_{\Gamma}}{N} \sum_{\boldsymbol{R} \boldsymbol{R}^{\prime}} e^{i \boldsymbol{k}^{\prime} \cdot \boldsymbol{R}^{\prime}} e^{-i \boldsymbol{k} \cdot \boldsymbol{R}} G_{\boldsymbol{R} \boldsymbol{R}^{\prime}}(\omega, \tilde{\boldsymbol{k}}),
$$

where $\boldsymbol{k}=\tilde{\boldsymbol{k}}+\boldsymbol{K}$ and $\boldsymbol{k}^{\prime}=\tilde{\boldsymbol{k}}+\boldsymbol{K}^{\prime}$. Note that we can make the substitution $\tilde{\boldsymbol{k}} \rightarrow \boldsymbol{k}=\tilde{\boldsymbol{k}}+\boldsymbol{K}$ in the Green's function $G_{\boldsymbol{R}^{\prime}}(\omega, \tilde{\boldsymbol{k}})$ on the right side, since $\tilde{\boldsymbol{k}}$ is a vector defined up to a vector $\boldsymbol{K}$ belonging to the reciprocal lattice of $\Gamma$. Note also that, since $\boldsymbol{K}$ and $\boldsymbol{K}^{\prime}$ may not be identical, the Green's function in Eq. (D14) is not diagonal in $\boldsymbol{k}=\tilde{\boldsymbol{k}}+\boldsymbol{K}$ and $\boldsymbol{k}^{\prime}=\tilde{\boldsymbol{k}}+\boldsymbol{K}^{\prime}$. For an element in the diagonal, the expression takes the following form, often called the periodization formula:

$$
G(\omega, \boldsymbol{k})=\frac{N_{\Gamma}}{N} \sum_{\boldsymbol{R} \boldsymbol{R}^{\prime}} e^{i \boldsymbol{k} \cdot\left(\boldsymbol{R}^{\prime}-\boldsymbol{R}\right)} G_{\boldsymbol{R} \boldsymbol{R}^{\prime}}(\omega, \boldsymbol{k}) .
$$

The periodization formula contains all the information needed to compute the density of states $\rho(\omega)$, as this only involves diagonal elements of the $\boldsymbol{k}$-representation of the Green's function,

$$
\rho(\omega)=-\frac{1}{\pi} \sum_{\boldsymbol{k}} \operatorname{Im} G\left(\omega+i 0^{+}, \boldsymbol{k}\right) .
$$

Moreover, the $\boldsymbol{k}$-resolved spectral function $A(\omega, \boldsymbol{k})$ also involves only elements on the diagonal. 
(a)

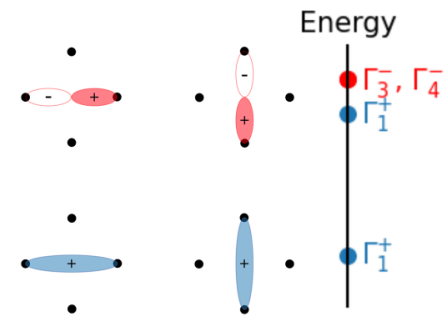

(b)

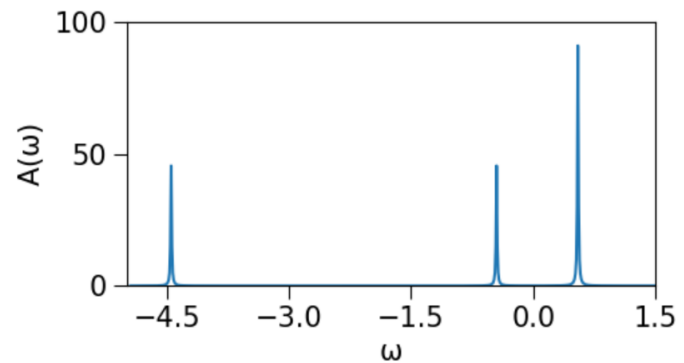

(c)

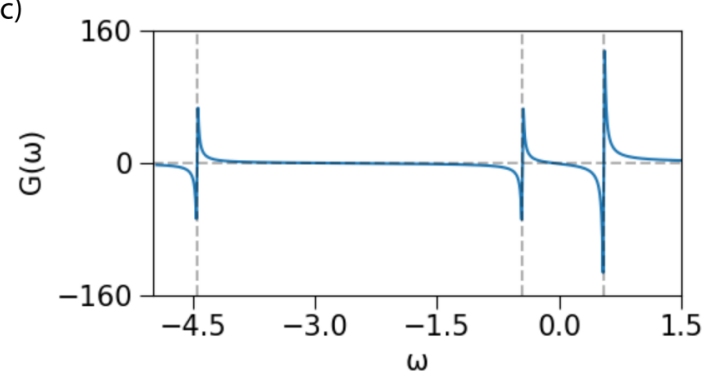

FIG. 14. Analysis of the single diamond in the AI phase with $t_{2} / t_{1}=1.5$. (a) Single-particle spectrum with $U=0$, where the bonding states whose combinations give rise to states transforming as $\Gamma_{1}^{+}$and antibonding states transforming as $\Gamma_{3}^{-}$and $\Gamma_{4}^{-}$are shown. Parts (b) and (c) show the traces of the single-particle spectral function and the Green's function computed with $U / t_{1}=1$, respectively.

Considering that the normalization of $\rho(\omega)$ reads $\int_{-\infty}^{\infty} d \omega \rho(\omega)=1$, the chemical potential $\mu$ is computed from $\rho(\omega)$ based on the following formula:

$$
\int_{-\infty}^{\mu} d \omega \rho(\omega)=1 / 2
$$

Equation (D17) can also be considered the equation defining $\mu$.

\section{APPENDIX E: ANALYSIS OF A SINGLE DIAMOND}

In this Appendix, we show the application of TQC to the Green's function of a single diamond, which may serve as a checkpoint to test our approach before tackling the periodic chain. We have calculated the single-particle Green's function and spectral function of the diamond with exact diagonalization at representative points within the AI, metal, and Mott phases. We have also computed the topological Hamiltonian and analyzed its spectrum in the framework of TQC.

\section{Atomic insulator}

The point representing the AI phase is located at $t_{2} / t_{1}=$ 1.5. The single-particle spectrum of the diamond with $U=0$ (a)

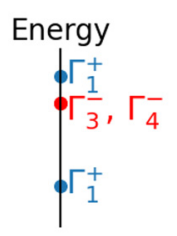

(b)

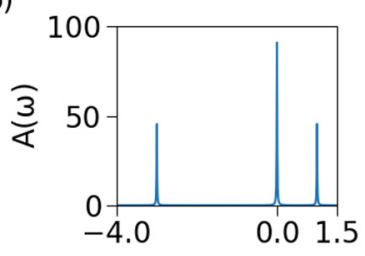

$\omega$

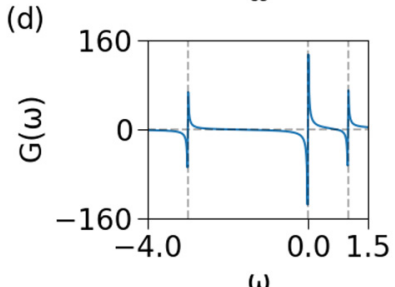

(c)

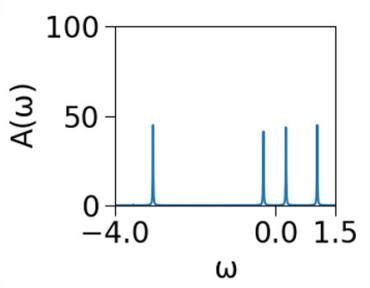

(e)

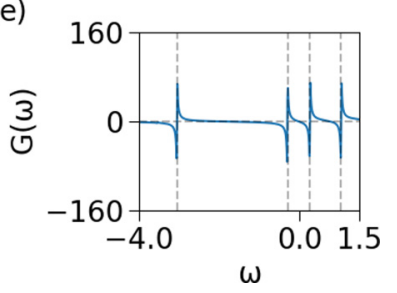

FIG. 15. Analysis of the diamond with $t_{2} / t_{1}=0.5$. Parts (a), (b), and (d) show the single-particle spectrum and traces of the spectral function and Green's function in the metal phase with $U=0$, while (c) and (e) contain the traces of the spectral function and the Green's function in the Mott phase with $U / t_{1}=1$.

is shown in Fig. 14(a), while Figs. 14(b) and 14(c) show the spectral function and the Green's function with $U / t_{1}=1$, respectively. The electronic structure with $U \neq 0$ is adiabatically connected to the $U=0$ limit, as the peaks in the spectral function and poles in the Green's function retain the features of the noninteracting electronic structure in Fig. 14(a). Accordingly, the lowest (highest) two levels in the spectrum of the topological Hamiltonian also have in correspondence wave functions transforming as irreps $\Gamma_{1}^{+}\left(\Gamma_{3}^{-}\right.$and $\left.\Gamma_{4}^{-}\right)$.

The single-particle spectrum in the AI phase (equivalently, the spectrum of the topological Hamiltonian) can be interpreted in terms of the quantum physics of the $\mathrm{H}_{2}^{+}$molecule, since the Hamiltonian $H_{\text {diam }}^{\mathrm{AI}}$ of the diamond in the AI phase is adiabatically connected to the following limit:

$$
H_{\text {diam }}^{\mathrm{AI}}=-t_{2} \sum_{\sigma} c_{1 \sigma}^{+} c_{3 \sigma}-t_{2} \sum_{\sigma} c_{0 \sigma}^{+} c_{2 \sigma}+\text { H.c. }
$$

To interpret the symmetry properties of the spectrum in Fig. 14(a), let us write the point group $D_{2 h}$ of the diamond as two slightly different coset decompositions:

$$
\begin{aligned}
D_{2 h}= & \left\{E, M_{z}, C_{2 x}, M_{y}\right\} \cup I\left\{E, M_{z}, C_{2 x}, M_{y}\right\}, \\
& \left\{E, M_{z}, C_{2 y}, M_{x}\right\} \cup I\left\{E, M_{z}, C_{2 y}, M_{x}\right\} .
\end{aligned}
$$

The first term on the right of Eq. (E1) is the Hamiltonian of a $\mathrm{H}_{2}^{+}$molecule formed by the two sites of the diamond that are on the $x$-axis in Fig. 1(a). Its ground state is dubbed a bonding state, and it is even with respect to both cosets in Eq. (E2a), thus it transforms as the irrep $\Gamma_{1}^{+}$. The excited state is known as an antibonding state due to the node positioned 
between both sites, and it is even with respect to the first coset in Eq. (E2a) and odd under the second, so it transforms as the irrep $\Gamma_{3}^{-}$. Both states are spatially distributed along the $x$-axis joining the sites.

The second term on the right of Eq. (E1) is also the Hamiltonian of a $\mathrm{H}_{2}^{+}$dimer, but composed by the two sites of the diamond that are out of the $x$-axis. Accordingly, its eigenstates spread on the direction normal to the $x$-axis. Whereas its bonding state is even with respect to all the symmetries in $D_{2 h}$ and transforms as $\Gamma_{1}^{+}$, the antibonding state is odd under the operations in the second coset of Eq. (E2b) and transforms as $\Gamma_{4}^{-}$.

The antibonding states coincide with the states transforming as $\Gamma_{3}^{-}$and $\Gamma_{4}^{-}$in the single-particle spectrum shown in Fig. 14(a) and they are degenerate in energy, as both terms in Eq. (E1) share the same coupling constant $t_{2}$. Considering that $t_{1}$ does not vanish in the whole AI phase, bonding states will hybridize, and as a result neither of the states transforming as $\Gamma_{1}^{+}$in Fig. 14(a) will be a pure bonding state, nor will they be degenerate in energy.

\section{Metal and Mott phases}

We choose $t_{2} / t_{1}=0.5$ with $U=0$ as the point representing the metallic phase. As can be seen in the single-particle spectrum in Fig. 15(a), the levels corresponding to irreps $\Gamma_{3}^{-}$ and $\Gamma_{4}^{-}$are not separated by a gap. Accordingly, the spectral function in Fig. 15(b) and the Green's function in Fig. 15(d) contain a peak and a pole at $\omega=0$, respectively. The spectrum of the topological Hamiltonian is also characterized by the absence of a finite gap between the second and third levels [61].

The single-particle spectral function computed with $U / t_{1}=1.0$ [Fig. 15(c)] shows the formation of Hubbard bands giving rise to a charge gap in the Mott phase, and each Hubbard band has in correspondence a pole in the Green's function [Fig. 15(e)]. The symmetric distribution of these poles around $\omega=0$ forces the Green's function to have eigenvalues that vanish at that frequency, giving rise to a singularity in the topological Hamiltonian $H_{T}=-G^{-1}(0)$.
[1] M. Z. Hasan and C. L. Kane, Colloquium: Topological insulators, Rev. Mod. Phys. 82, 3045 (2010).

[2] C. L. Kane and E. J. Mele, $Z_{2}$ Topological order and the Quantum Spin Hall Effect, Phys. Rev. Lett. 95, 146802 (2005).

[3] B. Andrei Bernevig, T. L. Hughes, and S.-C. Zhang, Quantum spin hall effect and topological phase transition in hgte quantum wells, Science 314, 1757 (2006).

[4] K. von Klitzing, The quantized hall effect, Rev. Mod. Phys. 58, 519 (1986).

[5] D. C. Tsui, H. L. Stormer, and A. C. Gossard, Two-Dimensional Magnetotransport in the Extreme Quantum Limit, Phys. Rev. Lett. 48, 1559 (1982).

[6] D. C. Tsui, Nobel lecture: Interplay of disorder and interaction in two-dimensional electron gas in intense magnetic fields, Rev. Mod. Phys. 71, 891 (1999).

[7] B. Bradlyn, L. Elcoro, J. Cano, M. G. Vergniory, Z. Wang, C. Felser, M. I. Aroyo, and B. A. Bernevig, Topological quantum chemistry, Nature (London) 547, 298 (2017).

[8] Z. Song, T. Zhang, and C. Fang, Diagnosis for Nonmagnetic Topological Semimetals in the Absence of Spin-Orbital Coupling, Phys. Rev. X 8, 031069 (2018).

[9] H. C. Po, A. Vishwanath, and H. Watanabe, Symmetry-based indicators of band topology in the 230 space groups, Nat. Commun. 8, 50 (2017).

[10] Z. Song, T. Zhang, Z. Fang, and C. Fang, Quantitative mappings between symmetry and topology in solids, Nat. Commun. 9, 3530 (2018).

[11] S. Rachel, Interacting topological insulators: A review, Rep. Prog. Phys. 81, 116501 (2018).

[12] Z.-C. Gu and X.-G. Wen, Tensor-entanglement-filtering renormalization approach and symmetry-protected topological order, Phys. Rev. B 80, 155131 (2009).

[13] F. Pollmann, A. M. Turner, E. Berg, and M. Oshikawa, Entanglement spectrum of a topological phase in one dimension, Phys. Rev. B 81, 064439 (2010).
[14] L. Fidkowski and A. Kitaev, Topological phases of fermions in one dimension, Phys. Rev. B 83, 075103 (2011).

[15] A. M. Turner, F. Pollmann, and E. Berg, Topological phases of one-dimensional fermions: An entanglement point of view, Phys. Rev. B 83, 075102 (2011).

[16] X. Chen, Z.-C. Gu, and X.-G. Wen, Classification of gapped symmetric phases in one-dimensional spin systems, Phys. Rev. B 83, 035107 (2011).

[17] T. Senthil, Symmetry-protected topological phases of quantum matter, Annu. Rev. Condens. Matter Phys. 6, 299 (2015).

[18] Q.-R. Wang and Z.-C. Gu, Towards a Complete Classification of Symmetry-Protected Topological Phases for Interacting Fermions in Three Dimensions and a General Group Supercohomology Theory, Phys. Rev. X 8, 011055 (2018).

[19] H. Song, S.-J. Huang, L. Fu, and M. Hermele, Topological Phases Protected by Point Group Symmetry, Phys. Rev. X 7, 011020 (2017).

[20] A. Kapustin, R. Thorngren, A. Turzillo, and Z. Wang, Fermionic symmetry protected topological phases and cobordisms, J. High Energy Phys. 12 (2015) 052.

[21] L. Fu, C. L. Kane, and E. J. Mele, Topological Insulators in Three Dimensions, Phys. Rev. Lett. 98, 106803 (2007).

[22] A. Altland and M. R. Zirnbauer, Nonstandard symmetry classes in mesoscopic normal-superconducting hybrid structures, Phys. Rev. B 55, 1142 (1997).

[23] C.-K. Chiu, J. C. Y. Teo, A. P. Schnyder, and S. Ryu, Classification of topological quantum matter with symmetries, Rev. Mod. Phys. 88, 035005 (2016).

[24] L. Fu, Topological Crystalline Insulators, Phys. Rev. Lett. 106, 106802 (2011).

[25] A. A. Soluyanov and D. Vanderbilt, Smooth gauge for topological insulators, Phys. Rev. B 85, 115415 (2012).

[26] M. G. Vergniory, L. Elcoro, C. Felser, N. Regnault, B. A. Bernevig, and Z. Wang, A complete catalogue of high-quality topological materials, Nature (London) 566, 480 (2019). 
[27] J. Zak, Symmetry Specification of Bands in Solids, Phys. Rev. Lett. 45, 1025 (1980).

[28] L. Michel and J. Zak, Connectivity of energy bands in crystals, Phys. Rev. B 59, 5998 (1999).

[29] L. Michel and J. Zak, Elementary energy bands in crystals are connected, Phys. Rep. 341, 377 (2001).

[30] H. Yao and S. A. Kivelson, Fragile Mott Insulators, Phys. Rev. Lett. 105, 166402 (2010).

[31] Y. Fuji, F. Pollmann, and M. Oshikawa, Distinct Trivial Phases Protected by a Point-Group Symmetry in Quantum Spin Chains, Phys. Rev. Lett. 114, 177204 (2015).

[32] V. Gurarie, Single-particle Green's functions and interacting topological insulators, Phys. Rev. B 83, 085426 (2011).

[33] Z. Wang and S.-C. Zhang, Simplified Topological Invariants for Interacting Insulators, Phys. Rev. X 2, 031008 (2012).

[34] Z. Wang and B. Yan, Topological hamiltonian as an exact tool for topological invariants, J. Phys.: Condens. Matter 25, 155601 (2013).

[35] C. Gros and R. Valentí, Cluster expansion for the self-energy: A simple many-body method for interpreting the photoemission spectra of correlated Fermi systems, Phys. Rev. B 48, 418 (1993).

[36] D. Sénéchal, D. Perez, and M. Pioro-Ladrière, Spectral Weight of the Hubbard Model Through Cluster Perturbation Theory, Phys. Rev. Lett. 84, 522 (2000).

[37] D. Sénéchal, D. Perez, and D. Plouffe, Cluster perturbation theory for Hubbard models, Phys. Rev. B 66, 075129 (2002).

[38] F. Manghi, Multi-orbital cluster perturbation theory for transition metal oxides, J. Phys.: Condens. Matter 26, 015602 (2013).

[39] I. P. McCulloch, Infinite size density matrix renormalization group, revisited, arXiv:0804.2509.

[40] M. P. Zaletel, R. S. K. Mong, and F. Pollmann, Topological Characterization of Fractional Quantum Hall Ground States from Microscopic Hamiltonians, Phys. Rev. Lett. 110, 236801 (2013).

[41] F. Becca and S. Sorella, Quantum Monte Carlo Approaches for Correlated Systems (Cambridge University Press, Cambridge, 2017).

[42] M. Capello, F. Becca, M. Fabrizio, S. Sorella, and E. Tosatti, Variational Description of Mott Insulators, Phys. Rev. Lett. 94, 026406 (2005).

[43] M. Capello, F. Becca, S. Yunoki, and S. Sorella, Unconventional metal-insulator transition in two dimensions, Phys. Rev. B 73, 245116 (2006).

[44] L. F. Tocchio, C. Gros, R. Valentí, and F. Becca, Onedimensional spin liquid, collinear, and spiral phases from uncoupled chains to the triangular lattice, Phys. Rev. B 89, 235107 (2014)

[45] F. Pollmann and A. M. Turner, Detection of symmetryprotected topological phases in one dimension, Phys. Rev. B 86, 125441 (2012).

[46] M. I. Aroyo, J. M. Perez-Mato, C. Capillas, E. Kroumova, S. Ivantchev, G. Madariaga, A. Kirov, and H. Wondratschek, Bilbao crystallographic server: I. Databases and crystallographic computing programs, Z. Krist.-Cryst. Mater. 221, 15 (2006).

[47] J. Perez-Mato, D. Orobengoa, E. Tasci, G. De la Flor Martin, and A. Kirov, Crystallography online: Bilbao crystallographic server, Bulg. Chem. Commun. 43, 183 (2011).
[48] Note that $C_{2 v}$ is a subgroup of $D_{2 h}$, where $D_{2 h}$ is the point group of an isolated diamond.

[49] Although the choice of restricting our analysis to positive hopping parameters means that we cannot study all insulator phases of the model, it gives us access to the part of the phase diagram containing the physics we are interested in, namely two different topological phases distinguished by symmetry and a Mott phase.

[50] Based on the similarity of the correlated insulator phases and the noninteracting $\mathrm{AI}$ and $\mathrm{OAL}$ phases, we may predict the possibility of the crossing between the lowest and last occupied bands of the topological Hamiltonian in the mentioned phases. Such an interplay of bands would not have any effect in the topological classification.

[51] U. Schollwöck, The density-matrix renormalization group in the age of matrix product states, Ann. Phys. 326, 96 (2011), January 2011 Special Issue.

[52] M. B. Hastings and T. Koma, Spectral gap and exponential decay of correlations, Commun. Math. Phys. 265, 781 (2006).

[53] K. Shiozaki, H. Shapourian, and S. Ryu, Many-body topological invariants in fermionic symmetry-protected topological phases: Cases of point group symmetries, Phys. Rev. B 95, 205139 (2017).

[54] H. Shapourian, K. Shiozaki, and S. Ryu, Many-Body Topological Invariants for Fermionic Symmetry-Protected Topological Phases, Phys. Rev. Lett. 118, 216402 (2017).

[55] G. E. Volovik, The Universe in a Helium Droplet (Oxford University Press, Oxford, 2003).

[56] G. E. Volovik, Topological invariant for superfluid 3he-b and quantum phase transitions, JETP Lett. 90, 587 (2009).

[57] G. E. Volovik, Topological invariants for standard model: From semi-metal to topological insulator, JETP Lett. 91, 55 (2010).

[58] Z. Wang, X.-L. Qi, and S.-C. Zhang, Topological order Parameters for Interacting Topological Insulators, Phys. Rev. Lett. 105 256803 (2010).

[59] Z. Wang, X.-L. Qi, and S.-C. Zhang, Topological invariants for interacting topological insulators with inversion symmetry, Phys. Rev. B 85, 165126 (2012).

[60] T. Mertz, K. Zantout, and R. Valentí, Statistical analysis of the Chern number in the interacting Haldane-Hubbard model, Phys. Rev. B 100, 125111 (2019).

[61] D. Lessnich, S. M. Winter, M. Iraola, M. G. Vergniory, and R. Valentí, Elementary band representations for the single-particle Green's function of interacting topological insulators, Phys. Rev. B 104, 085116 (2021).

[62] S. R. Manmana, A. M. Essin, R. M. Noack, and V. Gurarie, Topological invariants and interacting one-dimensional fermionic systems, Phys. Rev. B 86, 205119 (2012).

[63] Y.-Y. He, H.-Q. Wu, Z. Y. Meng, and Zhong-Yi Lu, Topological invariants for interacting topological insulators. II. Breakdown of single-particle Green's function formalism, Phys. Rev. B 93, 195164 (2016).

[64] Y.-Z. You, Z. Wang, J. Oon, and C. Xu, Topological number and fermion Green's function for strongly interacting topological superconductors, Phys. Rev. B 90, 060502(R) (2014).

[65] T.-J. Liu, M. A. Springer, N. Heinsdorf, A. Kuc, R. Valentí, and T. Heine, Tailoring fermion topological states in the fes (square-octagon) lattice using two-dimensional polymers, arXiv:2102.12324. 
[66] Y. Ishii, G. Sala, M. B. Stone, V. O. Garlea, S. Calder, J. Chen, H. K. Yoshida, S. Fukuoka, J. Yan, M.-H. Du et al., Magnetic properties of the Shastry-Sutherland lattice material $\mathrm{BaNd}_{2} \mathrm{ZnO}_{5}$, Phys. Rev. Mater. 5, 064418 (2021).

[67] M. I. Aroyo, A. Kirov, C. Capillas, J. M. Perez-Mato, and H. Wondratschek, Bilbao crystallographic server ii: Representations of crystallographic point groups and space groups, Acta Crystallogr. A62, 115 (2006).

[68] Web address of the Band Representations program BANDREP: www.cryst.ehu.es/cryst/bandrep.

[69] L. Elcoro, B. Bradlyn, Z. Wang, M. G. Vergniory, J. Cano, C. Felser, B. A. Bernevig, D. Orobengoa, G. Flor, and M. I. Aroyo, Double crystallographic groups and their representations on the Bilbao Crystallographic Server, J. Appl. Crystallogr. 50, 1457 (2017).

[70] S. R. White, Density-matrix algorithms for quantum renormalization groups, Phys. Rev. B 48, 10345 (1993).
[71] J. Hauschild and F. Pollmann, Efficient numerical simulations with Tensor Networks: Tensor Network Python (TeNPy), SciPost Phys. Lect. Notes 5 (2018).

[72] S. Sorella, Wave function optimization in the variational Monte Carlo method, Phys. Rev. B 71, 241103(R) (2005).

[73] R. P. Feynman, Atomic theory of the two-fluid model of liquid helium, Phys. Rev. 94, 262 (1954).

[74] R. Resta and S. Sorella, Electron Localization in the Insulating State, Phys. Rev. Lett. 82, 370 (1999).

[75] D. Sénéchal, An introduction to quantum cluster methods, arXiv:0806.2690.

[76] E. Dagotto, Correlated electrons in high-temperature superconductors, Rev. Mod. Phys. 66, 763 (1994).

[77] S. Pairault, D. Sénéchal, and A. M. S. Tremblay, StrongCoupling Expansion for the Hubbard Model, Phys. Rev. Lett. 80, 5389 (1998). 